\title{
12th Roche Diabetes Care Network Meeting: April 11-13, 2019, Copenhagen, Denmark
}

\author{
Christopher G. Parkin, MS, Christine Zepezauer, $\mathrm{MA}^{2}$ and Rolf Hinzmann, MD, $\mathrm{PhD}^{2}$
}

\begin{abstract}
A panel of international experts in the field of diabetes and diabetes technology met in Copenhagen, Denmark, for the 12th Roche Diabetes Care Network Meeting. The goal of these meetings is to share current knowledge, facilitate new collaborations, and encourage further research projects that can improve the lives of people with diabetes. Specific areas of interest included use of telemedicine and mobile health technologies, behavior change, patient-centered care, and multifactorial approaches to addressing all metabolic abnormalities associated with diabetes. The 2019 meeting covered a comprehensive scientific program and four keynote lectures.
\end{abstract}

Keywords: Continuous Glucose Monitoring (CGM), Self-Monitoring of Blood Glucose (SMBG), m-Health, Behavior, Closed loop.

\section{Opening Lecture}

News from the World of Diabetes

Satish Garg, University of Colorado Denver, Aurora, Colorado

\section{Background}

$\mathbf{H}$ EALTH CARE AND THE diabetes market are changing rapidly, driven by an aging population with deteriorating health. In the United States, $\sim 25 \%$ of all insulintreated diabetes patients are over the age of 65. Suboptimal diabetes control among a large percentage of people has prompted technological/scientific innovation and new metrics for assessing and monitoring diabetes status. Many of these innovations are prodding regulatory agencies to revise their requirements in assessing both safety and efficacy. Affordability of health care products and services, as well as access to health care, remains a key challenge moving forward.

\section{Challenges of diabetes prevalence}

More than 435 million people have diabetes, reflecting a nearly fourfold increase during the past four decades. The prevalence of diabetes continues to increase in the United States and now accounts for $\sim 14 \%$ of the population ( $\sim 50$ million), including those with diagnosed and undiagnosed diabetes. ${ }^{1}$

An estimated 200 million diabetes patients worldwide require insulin. Approximately $90 \%$ of these individuals have type 2 diabetes (T2D). It is known that $\sim 25 \%$ of patients with type 1 diabetes (T1D) will remain C-peptide positive, and an estimated $10 \%-20 \%$ of individuals diagnosed with T2D are actually misdiagnosed; they are positive for glutamic acid decarboxylase autoantibodies.

Meeting the health needs of diabetes patients who require insulin will continue to challenge health care systems, payers, and society due to several factors. A significant percentage of diabetes patients are not achieving their glycemic goals. ${ }^{2,3}$ With decreasing numbers of endocrinologists, more patients are being treated by primary care physicians, many of whom are inexperienced in insulin management. In addition, although insulin pumps and closed-loop systems have been proven effective in improving diabetes control, adoption of these technologies has been slow because they are not available and/or affordable for many patients. Worldwide, $<1 \%$ of people with insulin-requiring diabetes are using some sort of insulin pump therapy.

New approaches to care (e.g., telemedicine and coaching), new technologies that support better clinical management (e.g., digital decision support tools), and affordable tools/medications are needed to streamline care delivery and improve health outcomes for the masses. Otherwise, they will be left behind.

\footnotetext{
${ }^{1}$ CGParkin Communications, Inc., Henderson, Nevada.

${ }^{2}$ Roche Diabetes Care GmbH, Mannheim, Germany.
}

(c) Christopher G. Parkin, et al., 2020; Published by Mary Ann Liebert, Inc. This Open Access article is distributed under the terms of the Creative Commons Attribution Noncommercial License (http://creativecommons.org/licenses/by-nc/4.0/) which permits any noncommercial use, distribution, and reproduction in any medium, provided the original author(s) and the source are credited. 


\section{Going beyond glycated hemoglobin}

Although long considered the gold standard for assessing glycemic control over 2-3 months, the glycated hemoglobin $(\mathrm{HbA} 1 \mathrm{c})$ test has several limitations. Because each HbA1c level comprises a wide mean glucose range (e.g., 123$185 \mathrm{mg} / \mathrm{dL}$ for $7 \% \mathrm{HbA} 1 \mathrm{c})$ under- or overtreatment can occur.

Moreover, it does not reflect the occurrence and magnitude of inter- and intraday glucose excursions, limiting its usefulness in daily management.

One emerging concept is using time in ranges-within, above, and below - for glycemic assessment. Guidance from a recent consensus panel regarding use of these metrics with continuous glucose monitoring (CGM) data has recently been published along with recommendations for standardizing glucose data reporting, utilizing the Ambulatory Glucose Profile (AGP). ${ }^{4}$ In addition to presenting percentage of time in ranges, the AGP features a new metric, the glucose management indicator, which closely correlates with (calculated) HbAlc but utilizes data acquired over a much shorter time period ( 2 weeks).

\section{Adjunctive therapy for $T 1 D$}

Over the years, several adjunctive therapies for individuals with T1D have been explored. The only therapy to receive U.S. Food and Drug Administration (FDA) approval was pramlintide. However, this treatment was unpopular due to adverse gastrointestinal effects and increased hypoglycemia. The most promising adjunctive therapy is use of sodium/glucose cotransporter (SGLT) inhibitors: empagliflozin, dapagliflozin (SGLT-2), and sotagliflozin (SGLT-1\&2). These medications primarily focus on preventing reabsorption of glucose in the kidney.

Currently approved for use in T2D, these new medications have been evaluated extensively in T1D in three different research programs: Dapagliflozin Evaluation in Patients With Inadequately Controlled Type 1 Diabetes (DEPICT), ${ }^{5,6}$ inTandem, ${ }^{7-9}$ and Empagliflozin as Adjunctive to Insulin thErapy (EASE). ${ }^{10}$ In these studies, treatment with an SGLT2 or SGLT-1\&2 inhibitor was shown to improve glycemic control (both HbA1c reductions and increased time in range) and confer significant cardiometabolic and potential longterm renal benefits with no apparent increase in hypoglycemia in people with T1D. However, treatment was associated with increased incidence of diabetic ketoacidosis (DKA). Importantly, some of the DKA cases presented with nearnormal or slightly elevated blood glucose levels, referred to as euglycemic DKA (euDKA).

To address this concern, an international consensus panel recently published guidelines for mitigating DKA risk. ${ }^{11}$

\section{Emerging health problems}

The global prevalence of nonalcoholic fatty liver disease (NAFLD) has risen to $25 \%$. Prevalence of nonalcoholic steatohepatitis (NASH), a form of NAFLD but with accompanying hepatitis, is estimated to be $6.5 \%$ worldwide and $12 \%$ in the United States. NASH is associated with a high burden of metabolic comorbidities of diabetes, including obesity, hypertension, and dyslipidemia. Although many diabetes medications are being evaluated for possible use in treating these diseases, a recent report highlighted the effectiveness of Revita Duodenal Mucosal Resurfacing, a no- vel endoscopic procedure that uses heat to ablate the lining of the duodenum. With this procedure, investigators were able to achieve a $36 \%$ reduction in liver fat.

\section{Summary}

The rapidly evolving health care landscape is changing the way diabetes is managed. With increasing prevalence of diabetes and the growing number of insulin-requiring patients, metrics beyond $\mathrm{HbA} 1 \mathrm{c}$ are needed to more accurately assess glycemic status and improve outcomes. Developing adjunctive therapies that help patients control weight and reduce hypoglycemia risk should continue to be a priority in current and future research.

\section{Session A: Keynote Lecture}

\section{Diabetes Is More Than Elevated Glycated Hemoglobin: What We Need Beyond This Surrogate Marker to Improve Long-Term Outcomes}

Stephan Jacob, Gemeinschaftspraxis Prof. Dr. Stephan Jacob und Dr. Frohmut Jacob Diabetes \& Endocrinology, Villingen-Schwenningen, Germany

\section{Background}

The increasing prevalence of T2D and its associated complications threatens to cripple health care systems, worldwide. Individuals with $\mathrm{T} 2 \mathrm{D}$ have a higher rate of complications than those with T1D. Life expectancy is shortened by 6 years in individuals with diabetes and reduced by 12 years if they have suffered a myocardial infarction. The primary focus of T2D management has traditionally been glucose control. More than $80 \%$ of T2D patients present with the full picture of metabolic syndrome, a condition that impacts several organ systems in the body.

\section{Impact of glycemic control}

$\mathrm{HbA} 1 \mathrm{c}$ is the most common endpoint used to assess the relationship between glycemic control and the microvascular/macrovascular complications of diabetes. There are clear associations between glycemic control (as assessed by $\mathrm{HbA1c}$ ) and diabetes complications. However, most intervention studies aiming to lower HbAlc failed to reduce morbidity and mortality. Although studies have shown HbA1c improvements when comparing intensive interventions to usual care, ${ }^{12-15}$ in many of these assessments, usual care was suboptimal (e.g., Veterans Administration Diabetes Trial [VADT]). ${ }^{15}$ Because intensive interventions to lower HbA1c do not translate into reductions in all-cause mortality for several years, it is questionable whether the benefits of good glycemic control outweigh the added burden on patients.

\section{Is HbA1c the correct measure of diabetes control?}

Although several factors may have impacted the effectiveness of intensive therapies in reducing mortality (e.g., clinical inertia), studies suggest that $\mathrm{HbA} 1 \mathrm{c}$ may be the wrong clinical target, given the side effects of intensive management (e.g., weight gain and hypoglycemia), which are risk factors for cardiovascular $(\mathrm{CV})$ events. This is clearly demonstrated in the LEADER study, which showed a strong 
association between severe hypoglycemia and all-cause mortality. ${ }^{16}$ Moreover, because HbA1c only reflects the mean glucose load during the previous 3 months, it provides no information about intra- and interday glucose excursions.

\section{Lack of safety studies for CV risk}

Most of the early diabetes medications were never evaluated for CV safety in randomized controlled trials (RCTs). The only exceptions are acarbose and glargine. However, studies of the newer medications, including thiazolidinediones, dipeptidyl peptidase-4 (DPP-4) inhibitors, SGLT (SGLT-2, SGLT-1\&2) inhibitors, and glucagon-like peptide (GLP-1) agonists, have been extensively assessed for CV risk. In a recent study that looked at CV risk associated with adding a second medication to type 2 patients treated with metformin, O'Brien reported that the newer medications conferred a significantly lower risk for incidence of a first major CV event compared with sulfonylurea or glargine treatment. ${ }^{17}$ Moreover, in the EMPA-REG study, investigators reported significant reductions in $\mathrm{CV}$ events in T2D patients treated with empagliflozin (SGLT-2 inhibitor). ${ }^{18}$ Other SGLT-1/SGLT-1\&2 studies have also shown reductions in risk for renal disease.

\section{Multifactorial approach to diabetes management}

Although glucose control remains an important goal of therapy, a multifactorial approach is needed to reduce the complications of diabetes. As demonstrated in the STENO-2 trial, individuals who were able to achieve good control in glucose, blood pressure, and lipids had sustained beneficial effects in reducing vascular complications and all-cause mortality rates at 21.2 years of follow-up. ${ }^{19}$ In a populationbased study that looked on five clinical measures-glucose, blood pressure, lipids, albuminuria, and smoking-investigators found that individuals with $\mathrm{T} 2 \mathrm{D}$ who were well controlled in all of these measures had the same risk for $\mathrm{CV}$ disease and mortality as individuals without diabetes. ${ }^{20}$ These studies suggest that if individuals have elevated $\mathrm{HbAlc}$ and high CV risk, a GLP-1 receptor agonist or SGLT-2/SGLT-1\&2 inhibitor should be considered for treatment intensification.

\section{Summary}

Although lowering glucose has long been the primary focus in managing T2D, achieving established HbA1c goals has not proven effective in reducing mortality. Optimal diabetes management should involve a multifactorial approach that includes lifestyle interventions to control weight and early initiation of combination therapy, using multiple agents with different and complementary mechanisms of action to address all metabolic abnormalities associated with T2D. Good diabetes management should be more than just glucose control. It is time for a paradigm shift.

\section{Session B/Lecture 1}

\section{Why and When We Need Randomized Controlled Trials and How We Can Improve Them} John Pickup, King's College London, London, United
Kingdom

\section{Background}

RCTs are designed to minimize bias by randomly distributing patient characteristics and other confounders that may affect outcome to the intervention and control groups. This helps to ensure that the difference in outcome is only due to the intervention. Although RCTs are generally considered to be the gold standard of research, a potent marketing tool for industry, and the basis for cost-effectiveness calculations and regulatory approval, it has long been recognized that the perfect RCT is unattainable.

\section{Main problems with RCTs}

RCTs are needed when there is a gradual or small to moderate effect in a fluctuating condition, and when there are conflicting observational data. However, RCTs have several limitations that can impact their value in assessing the value of the interventions studied and result in misleading interpretation and reporting of findings. The main problems with RCTs are poor trial design, operational issues, and organizational issues.

Poor study designs may involve a number of factors: absence of blinding (patient and investigator), which can lead to bias; underpowering (small sample size); use of inappropriate, obsolete, or poorly executed treatments; poor external validity (study participants not from a population of interest); inappropriate endpoints; and inadequate time for accurate assessment. An example is a 2009 meta-analysis of severe hypoglycemia in patients treated with multiple daily insulin injections (MDI) versus continuous subcutaneous insulin infusion (CSII) therapy. ${ }^{21}$ Among the 12 trials included in the analysis, one trial was severely underpowered $(n=17)$, two trials were too short (3 and 4.5 months), two trials had significant baseline imbalances, one trial had no washout between crossover, and one trial had no run-in period. None of the trials had any allocation concealment, and one trial had multiple discrepancies between the individual patient data results and the results published in the actual article.

Operational issues such as slow and inadequate recruitment are also common problems when conducting RCTs. For example, $61 \%$ of internationally registered trials in women's health did not meet the planned sample size, and 69\% of U.K. trials funded by the Medical Research Council (MRC) and the Health Technology Assessment Program did not reach the recruitment target. The median duration of industrysponsored trials was increased by $70 \%$ over the planned time by delayed enrollment.

Organizational issues such as complexity, bureaucratic obstacles, and time requirements are key challenges. It is difficult to stay current with rapidly evolving technologies. For example, trials using older generation CGM devices can be outdated even before they are published. Cost is also an important concern. The average cost of a phase 3 RCT has grown to more than 30 million U.S. dollars in recent years. One way to reduce costs is to use "adaptive" trial designs. These types of studies utilize prospective, planned modifications of the trial protocol based on accumulating data. This has the potential to reduce costs and study duration and increase the success of the study. Another approach is to utilize sample size reassessment to ensure adequate power by increasing the number of subjects recruited and then changing 
the allocation ratio, favoring enrollment of a more promising treatment. Changing the eligibility criteria and enrolling more patients who are likely to benefit also increase efficiency. Finally, the study may be stopped early when efficacy, safety, or futility has been demonstrated.

\section{Summary}

One way to increase the validity and efficiency of RCTs is to use "pragmatic" clinical trials, which deliberately include a broader demographic or clinical range of patients (e.g., adults and children) and different types of study site and skills (e.g., community and nonacademic centers).

Real-world, observational studies can then be conducted to assess the effectiveness of an intervention under real-world conditions. Use of both approaches in combination makes it possible to describe an evidence-based pathway, looking first at efficacy and then at effectiveness.

Several issues must be considered to ensure the quality of study designs. It is important to make sure the study is sufficiently powered with long duration to determine the significance of results within a population of interest. Factors that are likely to minimize recruitment issues are telephone reminders to nonresponders, financial incentives for patients, open rather than blinded allocation, and opt-out procedures for potential recruits. Use of pragmatic study designs considered when demonstrating external validity is deemed important. Comparators should be current and appropriate. Investigators should strive to reduce bias by using blinding, when possible, and concealment of allocation to maximize recruitment and efficiency. Adaptive study designs should be considered to further reduce time and costs.

\section{Session B/Lecture 2}

\section{How Real-World Evidence Can Complement Randomized Controlled Trials}

\section{Kamlesh Khunti, University of Leicester, Leicester, United} Kingdom

\section{Overview}

The hierarchy of evidence grading for evidence-based guidelines considers meta-analyses of RCTs the highest level of evidence (Level 1a), closely followed by well-designed RCTs (Level 1b). Consensus recommendations and reports are considered the lowest level of evidence (Level 4). Although RCTs provide insights regarding the extent to which an intervention is more positive than negative under ideal conditions, real-world evidence (RWE) measures the extent to which an intervention does more positive than negative under usual circumstances in clinical practice.

There is an increasing interest in the use of RWE. In a meta-analysis of RWE trials published in 2000 in the New England Journal of Medicine, ${ }^{22}$ investigators concluded that the results of well-designed observational studies do not systematically overestimate the magnitude of effects of treatment compared with those in RCTs on the same topic.

Real-world data (RWD) are also very important when investigating adverse events, especially events that are rare. A recent meta-analysis, which compared adverse events derived from RCTs versus observational studies, concluded that there is no difference, on average, in the risk estimate of adverse effects in meta-analysis of RCTs versus observational studies.

\section{$R W D$ and RWE}

RWD are data collected outside of the constraints of an RCT. Sources of RWD include claims databases, medical records, patient registries, and health surveys, as well as supplemental data from RCTs. In addition, an increasing number of outcomes are being assessed using RWD, including quality-of-life measures, clinical outcomes, psychological factors, financial burden, adherence, and persistence.

There are distinct differences between RCTs and RWE studies. Whereas RCTs provide internal validity, RWE provides generalizability to larger, more diverse populations. Although there are many differences between the two study types, a key difference is cost. The cost of a cardiovascular outcome trial (CVOT) now averages \$150-\$160 million.

RWE is now becoming important for prescribers, payers, patients, and regulatory agencies. However, it is still uncertain whether the regulatory agencies will issue recommendations and product approvals based on RWE. In diabetes, the only example of a regulatory clearance based on RWE was use of metformin in individuals with heart failure. These individuals were excluded from metformin clinical trials due to the potential for lactic acidosis. However, reviews of observational studies showed that the adjusted risk ratio for metformin compared with other treatments favored metformin for all-cause mortality.

\section{Low representation of patients in RCTs}

RCTs often exclude elderly patients, patients with comorbidities, and those taking multiple medications. However, because only $17 \%$ of all individuals with diabetes meet these criteria, the applicability of RCT results in real-world clinical practice is questionable because most patients are not represented in those trials. Moreover, the evidence provided by RCTs is often not being put into practice, even among patients who would have met RCT inclusion eligibility. In addition, strict eligibility requirements in RCTs often exclude patients who would benefit most from the intervention and, thus, the magnitude of improvement is, by default, underreported. Examples of this are studies that put restrictive upper limits for baseline HbA1c. It is known that HbA1c reductions are generally much greater in those with high HbAlc levels at baseline. The same is true when assessing the impact of medications (e.g., GLP-1 receptor agonists) on weight loss. Patients with $>40$ body mass index (BMI) tend to lose more weight than those with lower BMIs, however, these patients are often excluded from studies. ${ }^{23}$ Importantly, when assessing hypoglycemia, the event rates reported in RCTs are significantly lower than those reported in global observational trials, which include large, diverse populations that are not represented in RCTs. Although RCT data have shown significant reductions in hypoglycemia with the use of new glucoselowering medications, a recent observational study from the United Kingdom showed no improvement in hospital admissions for hypoglycemia despite these new medications.

\section{Improving applicability to RWE from RCTs}

Confirmation of findings from RCTs in the real-world setting is important. For example, a 2010 RCT by Zoungas 
et al. showed an association between severe hypoglycemia and the risks of macrovascular or microvascular events and death among 11,140 patients with T2D. ${ }^{16}$ This assessment was later repeated in a real-world, retrospective cohort study of 13,682 individuals with T1D and T2D from a large database. Findings from this analysis confirmed the findings from the earlier RCT but also extended the findings to show that there was no difference in risk between diabetes groups. It was also found that the risk of $\mathrm{CV}$ events in individuals in both diabetes groups with established CV disease was significantly higher than in those without CV disease; a finding not reported in the earlier RCT.

Another example is the 10-year follow-up study of the U.K. Prospective Diabetes Study (UKPDS) cohort, which showed the "legacy effect" on reducing microvascular and macrovascular complications by improving glycemic control early, even if those improvements are not sustained. This effect was confirmed in a real-world analysis that assessed the impact of early intensification versus not intensifying therapy on increased incidence of retinopathy and CV complications.

When applied to comparative effectiveness studies, observational analyses further demonstrate how RCTs do not adequately represent real-world populations. For example, the three major SGLT-2 inhibitor trials demonstrated benefit in reducing major adverse cardiac events in patients with atherosclerotic CV disease. However, only two of the studies looked at patients without $\mathrm{CV}$ disease but with multiple risk factors. These studies showed the same beneficial effects. When looking at hospitalizations for heart failure, the beneficial effects of SGLT-2 inhibitor treatment are shown mainly in patients who are renally compromised. Although these findings are relevant to individuals with $\mathrm{CV}$ and/or renal disease, the majority of individuals with diabetes do not have $\mathrm{CV}$ disease in the real-world setting. Moreover, considerably more patients enrolled in the study were being treated with protective therapies (e.g., antihypertensives and statins) compared with those in the real world.

\section{Summary}

Well-designed, real-world studies complement RCTs. However, it is important to assess the totality of the available evidence and consider the types of RWD used and where they come from. More pragmatic trials are needed, with greater representation from the real-world population to more fully answer specific research questions and reduce costs. With growing awareness of the strengths and limitations of real-world studies, methodologies for conducting these studies are improving.

\section{Session C/Keynote Lecture}

\section{Reversing Type 2 Diabetes Is Possible: An Overview of the Evidence and the Limitations}

Michael Lean, University of Glasgow, Glasgow, United Kingdom

\section{Background}

$\mathrm{T} 2 \mathrm{D}$ is a chronic, progressive disorder that requires lifelong treatment. Despite clinical guidelines and use of medications to lower HbA1c, blood pressure, and lipids, current approaches to diabetes management have not improved life expectancy in individuals with T2D. At age 55-the average age for diagnosis - an individual with T2D will lose 5-6 years of life.

Weight gain/obesity is a primary driver of T2D development and progression. Bariatric surgeons have claimed diabetes remissions (up to 75\%) by weight loss for many years, which has now been proven in RCTs. In 2008, Dixon et al. published findings from the first RCT investigating the impact of bariatric surgery on weight loss and diabetes remission. ${ }^{24}$ Results showed $83 \%$ remission with $>15 \mathrm{~kg}$ weight loss.

However, bariatric surgery is expensive and only accessible to small numbers of people. Moreover, it has frequent complications. Approximately one in four patients who undergo bariatric surgery requires additional surgery to correct problems, and almost all patients require lifelong medical support and micronutrient supplementation.

\section{Efficacy of dietary interventions}

An early T2D study demonstrated that $>15 \mathrm{~kg}$ weight reduction through dietary interventions improved life expectancy to that of individuals who had never had diabetes. Although similar results can be achieved through very lowcalorie diets (VLCDs), many individuals eventually regain the weight they had lost. However, a more recent study showed that VLCDs were not necessary; weight loss in individuals ingesting $415 \mathrm{kcal} /$ day was the same as in those consuming $810 \mathrm{kcal} / \mathrm{day}$ at 16 weeks. This finding suggested that neither study group was adherent to diets. The investigators then initiated support programs to see if long-term weight loss could be improved. Although supervised exercise interventions showed no change, use of a structured program of meal replacements resulted in significant improvements in weight maintenance.

In a feasibility study in Scotland, investigators evaluated use of a weight management program in primary care. ${ }^{25}$ The goal was weight loss of $>15 \mathrm{~kg}$ at 12 months in 91 patients with $\mathrm{BMI} \geq 40 \mathrm{~kg} / \mathrm{m}^{2}$. Patients commenced a micronutrientreplete $810-833 \mathrm{kcal} /$ day for a planned 12 weeks or $20 \mathrm{~kg}$ weight loss (whichever was the sooner), with structured food reintroduction and then weight-loss maintenance. At study end, one-third of patients had achieved and maintained weight loss of $\geq 15 \mathrm{~kg}$. This intervention, which became the model for the National Health Service in Scotland, demonstrated virtually identical efficacy in weight loss compared with bariatric survey at $<10 \%$ of the per-patient cost.

\section{Diabetes Remission Clinical Trial}

The Diabetes Remission Clinical Trial (DiRECT) was designed to assess diabetes remissions in people with noninsulin-treated T2D. ${ }^{26}$ The intervention was a nonsurgical, weight management program, delivered within routine primary care settings located in deprived areas in Scotland and Northern England. ${ }^{27}$ Patients, age 20-65 years with average BMI $35 \mathrm{~kg} / \mathrm{m}^{2}$, were randomized to either the weight management program $(n=149$, intervention) or usual bestpractice care ( $n=149$, control).

The intervention comprised total liquid diet replacement (825-853 kcal/day formula diet) during the first 12 weeks, with 20- to 30-min appointments with a trained local dietitian or nurse at biweekly intervals. During the next 12-18 weeks, investigators initiated stepped transition to a food-based diet. 
This phase was particularly difficult for many patients because they were returning to the food and eating habits that had initially caused their diabetes. Consultations with dietitians/nurses at biweekly intervals were used to support patients through this transition. Weight-loss maintenance was initiated during weeks 19-104, during which time patients were advised to follow a food-based diet and provided a personalized energy prescription to support weight stabilization and prevent weight gain.

At weeks 12-20, investigators observed a mean weight loss of $14.5 \mathrm{~kg}$ in intervention patients. However, patients eventually regained some of this weight over the course of the study. At 12 months, the average weight loss was 11.6 and $8.8 \mathrm{~kg}$ at 24 months. Almost half (46\%) of intervention patients were in remission at 12 months and $36 \%$ at 24 months. Among intervention patients who maintained $>10 \mathrm{~kg}$ weight loss, the remission rate was $64 \%$ at 24 months. Remission rates among control patients remained low at 12 months (4\%) and 24 months (3\%).

Subanalyses of intervention patients in remission showed significant reductions in liver fat and very low-density lipoprotein (VLDL-1) production rates as well as improved first-phase insulin response. Investigators also observed significant reductions in $\mathrm{HbA1c}$ and blood pressure with fewer medications compared with control patients. Other CV risk factors and quality of life also improved. The only safety signal in DiRECT was in the control arm, which showed significantly more serious adverse events in the second year than the intervention group. The cost of providing the intervention program was less than half the average health care cost of conventional diabetes treatment. Findings from DiRECT demonstrate that remission can be achieved with $>10 \mathrm{~kg}$ weight loss, which should be a primary aim of diabetes care.

\section{Summary}

T2D is a disease of ectopic fat accumulation but not necessarily permanent. A structured weight management that can sustain remission to a nondiabetic state is now a practical and important treatment target, with great personal benefits. Future research must focus on improving the achievement and maintenance of sufficient weight loss and must widen the scope to include racial and ethnic groups whose diabetes has different characteristics.

The major challenge is to quickly allocate the funds and resources needed to support diabetes remission services. Long-term data on the life-shortening complications of diabetes are needed.

\section{Session D/Lecture 1}

\section{What Is and Why Do We Need Value-Based Health Care? Answers and Examples from the Field of Diabetes}

Tove Holm-Larsen, Pharma Evidence, Copenhagen, Denmark

\section{Background}

Health care costs are increasing throughout the Western world. These increases are driven by the growing number of elderly citizens, the continuing obesity epidemic, and new developments within personalized medicine that are turning broad disease areas, such as breast cancer, into numerous, highly specific rare diseases that demand new technologies for diagnosis and treatment. However, Western societies are experiencing challenges in meeting these increasing costs.

\section{Why value-based health care is needed}

In most countries, the traditional approach to providing care is institution-based, which is driven primarily by economics and budgeting issues. Although this approach allows for more efficient planning for capital expenditures (e.g., large diagnostic equipment), it does not incentivize improvements in the quality of care.

Activity-based health care approaches focus primarily on bureaucratic and staff activity (e.g., excessive meetings) with little incentive to provide patient care. The result is extended waiting periods for health care services and minimal incentives for improving outcomes.

Value-based health care is an approach to care delivery that focuses on the person rather than simply treating illness. For people with a chronic illness, such as diabetes, valuebased health care supports the use treatments that are personalized to best fit the clinical needs of the individual. The idea being that even if the treatment is more expensive than other options, using the most appropriate therapy will more rapidly and effectively improve diabetes control, thereby reducing the risk and much greater financial costs of the longterm complications of diabetes. However, value-based health care reaches even farther back into the disease process, promoting and facilitating diabetes prevention, leading to even greater cost savings.

Although institutions that have adopted value-based health care are highly incentivized to improve outcomes across the entire continuum of care, cost-efficiencies are often difficult to measure. Nevertheless, this approach confers many benefits to all stakeholders. Patients benefit by lower costs and better outcomes. Providers have higher patient satisfaction rates and better care efficiency, and they are incentivized financially to promote prevention. For payers, the approach provides stronger cost controls with reduced risk. Suppliers have greater alignment of prices with patient outcomes, and society benefits from reduced health care spending and overall better health.

\section{Changing the paradigm}

Initiating value-based health care requires rethinking the health care system. Patient care must provide patients with clinical-grade feedback on their status and clear instructions regarding their treatment regimens. Services and care must be closely coordinated using real-time data. Patient outcomes and engagement must be measurable. Importantly, the clinical environment must foster proactive collaboration between health care providers and patients.

There are six overall steps for ensuring successful implementation of value-based health care: (1) organize treatment into integrated units; (2) calculate results and costs on a per-patient basis; (3) calculate one total payment per overall treatment; (4) integrate previously siloed health care services/sectors; (5) establish community centers that facilitate prevention and support therapy adherence; and (6) utilize 
integrated, well-functioning information technology

solutions to facilitate information and data sharing.

The underlying principles of value-based health care include the following: payment for outcomes, not processes; risk-sharing among stakeholders (providers, payers, and pharmaceutical/device companies) to ensure fairness for both the health care and private sectors; and getting the right decision makers to agree on terms. Importantly, implementation must be patient-centered and functional no matter the number of patients served. Value calculation must be continuous and visible to all stakeholders. The invoicing structure must be functional for all parties. Importantly, reimbursement must be based on a common measure; higher reimbursements when outcomes exceed defined acceptable levels, lower when outcomes fall short of those levels.

\section{Summary}

Value-based health care promotes true patient centrism by keeping the focus on individual patients and increasing the incentives for follow-up and risk reduction. The approach offers the potential for significant cost savings by prioritizing high-risk patients and providing additional leverage when negotiating medication costs. Although initiating valuebased health care can be difficult, innovative digital solutions can support the transition.

\section{Session D/Lecture 2}

\section{Connecting the Dots for Outcome-Based Diabetes Management}

Kamlesh Khunti, University of Leicester, Leicester, United Kingdom

\section{Background}

Evidence-based guidelines are developed to evaluate the variations in care, assess efficacy and effectiveness gaps, both in population-level interventions and pharmaco-effectiveness interventions. The guidelines then recommend methods to improve the efficacy-to-effectiveness gap-the difference between results reported in RCTs and those revealed in realworld studies.

For implementation of research, outcomes are defined as the effects of deliberate purposefulness of actions to implement new treatments, practices, and services. The outcomes serve three functions: as indicators of the implementation success; as proximal indicators of implementation processes; and as key intermediate outcomes in relation to the system or clinical outcomes in treatment effectiveness and quality-ofcare research.

A number of RCTs have shown that glucose-lowering therapies improve glycemic control and reduce microvascular and macrovascular complications, especially when used aggressively early in the disease. ${ }^{12,13,28,29}$ Findings from these studies were then translated into guidelines, which are continually updated. The most recent European Association for the Study of Diabetes (EASD)/American Diabetes Association (ADA) consensus guidelines for management of T2D include an algorithm for medication intensification. However, an item often missed is text included in the graphics, which cautions clinicians to reassess patients and modify treatment every 3-6 months to avoid clinical inertia. ${ }^{30}$

\section{Efficacy-to-effectiveness gap}

Despite the availability of high-quality evidence and international evidence-based guidelines, many patients fail to achieve risk factor targets in clinical practice. As reported in the GUIDANCE study, $90 \%$ of the 7597 patients with diabetes included in the trial had HbA1c measurements; however, only $38 \%-70 \%$ (depending on the country) were achieving HbA1c level $<7 \% .^{31}$ Data from the United Kingdom, which includes electronic health records on over 3 million people with diabetes, showed that only $50 \%$ of patients are meeting all eight process measures for T2D management: smoking cessation, BMI reduction, foot examinations, urine albumin, creatinine, cholesterol, blood pressure, and HbA1c. Less than $40 \%$ achieved all three treatment targets for $\mathrm{HbA} 1 \mathrm{c}$, blood pressure, and lipids.

A number of factors, specifically therapeutic inertia and adherence to therapies, have been implicated in this efficacyto-effectiveness gap. Reasons behind the efficacy-toeffectiveness gap include population differences, biological differences (e.g., variability in drug response), and behavioral differences, which may be the most influential.

Diabetes prevention trials illustrate how population differences can impact outcomes in randomized trials but then confound the value/feasibility of the interventions when translated into real-world practice. For example, a 2007 meta-analysis concluded that lifestyle changes and pharmacological interventions can reduce the progression of T2D in those with impaired glucose tolerance. ${ }^{32}$ However, a systematic review of real-world diabetes prevention interventions showed significant variation in effect size. ${ }^{33}$ Although it was possible to achieve weight reductions, very few studies have demonstrated diabetes prevention. In addition, in all of the studies considered, participants were highly motivated to reduce their risk, which is not reflective of the general population. Moreover, the interventions were frequent, time-intensive, and not feasible in most real-world settings.

A case study of the Diabetes Prevention Program showed an exponential decline in use of the intervention (50\%), staff participation (25\%), patients will to participate $(12.5 \%)$ and then follow the regimen correctly $(6.2 \%)$. Among these patients, $3.2 \%$ benefited from the intervention and only $1.8 \%$ continued to benefit after 6 months.

\section{Inertia}

Failure to advance therapy or deintensify therapy when appropriate is a primary contributor to the efficacy-toeffectiveness gap. Deintensifying therapy in older adults is particularly important. A 2013 retrospective study looked at inertia in poorly controlled T2D. In individuals treated with one oral agent, the median time to intensification to two agents was 2 years, 7.2 years to intensify from two to three orals, and 6 years to intensify from two to three orals to insulin despite persistent $\mathrm{HbA} 1 \mathrm{c}$ levels of $\geq 7.5 \% .^{34}$ Other studies have shown similar delays in insulin initiation and titration. One of the significant consequences of failure to intensify therapy is that patients who fail to achieve their 
target HbA1c within the first 3 months are less likely to reach their target at 24 months.

\section{Adherence}

Suboptimal adherence is also a driver of the efficacy-toeffectiveness gap. In RCTs assessing GLP-1 receptor agonist treatment, $\mathrm{HbA} 1 \mathrm{c}$ reductions were consistently $\sim 1.0 \%$. However, an analysis by Carls et al. found that reductions were only $0.52 \%$ in real-world settings. ${ }^{35}$ Statistical modeling revealed that $\sim 75 \%$ of the gap between the efficacy and effectiveness of GLP-1 receptor agonist treatment was due to poor adherence. These findings were supported in a systematic review of 8 observational studies in which the mean rate of poor adherence was $37.8 \% .^{36}$

\section{Closing the gap}

There are several barriers to treatment adherence, including patient perceptions about certain therapies, impaired quality of life, concern about side effects, complexity of regimens, and financial restrictions. ${ }^{37}$ Although interventions to educate and support patients can play an important role in addressing these barriers, closing the efficacy-to-effectiveness gap will require tools and strategies to reduce inertia, such as developing more meaningful quality measures, making more effective use of information systems, providing personal feedback to clinicians, and streamlining access to patient data, presented in formats that can be easily interpreted and acted upon.

\section{Summary}

Early preventative interventions to achieve optimal diabetes control are associated with long-term clinical outcomes. However, there are significant efficacy-to-effectiveness gaps in the real-world setting and many barriers to translating evidence into real-world practice. Addressing these barriers will require a number of different solutions. However, the key is individualizing not just the therapies but the interventions to the patients as well.

\section{Session D/Lecture 3}

\section{Digitally Enabled Diabetes Management Facilitates State-of-the Art Personalized Care}

\section{Valérie Babinsky, Roche Diabetes Care, Vienna, Austria}

\section{Background}

Despite advances in medications, glucose monitoring technologies, and insulin delivery systems, only 50\%-70\% of individuals with diabetes are achieving their glycemic targets, and $<9 \%$ are achieving combined targets (glucose, blood pressure, and lipids) in real-world registries. This image differs in many cases substantially from the efficacy of modern therapies proven in large-scale RCTs. This phenomenon, the efficacy-to-effectiveness gap, is driven by multiple deficiencies in current models of diabetes care, including the setup of the clinical care system and the way people with diabetes and treating physicians independently and collaboratively treat diabetes.

\section{Drivers of the efficacy-to-effectiveness gap}

A primary driver of suboptimal diabetes control is failure to intensify therapy, which is referred to as clinical inertia. ${ }^{38}$ Described reasons for clinical inertia to occur include insufficient time to adequately assess and counsel patients in the routine clinical visits, scattered or poorly structured clinical information, financial and human resource constraints, and in many cases, the lack of a holistic process for patient-centric care. Furthermore, one of the most impactful contributors to the efficacy-to-effectiveness gap is poor patient adherence to therapy. ${ }^{35}$

The World Health Organization defines adherence as "the extent to which a person's behavior-taking medication, following a diet, and/or executing lifestyle changes-corresponds with the agreed recommendations from a health care provider." Adherence to therapy is indirectly proportional to disease burden and is driven by several factors, which can be categorized as either intentional (e.g., to avoid medication side effects and complexity of the regimen) or unintentional (e.g., forgetfulness and difficulty changing lifestyle behaviors). ${ }^{39}$ Although the causes of clinical inertia and poor adherence are multifactorial, a major contributor to both is lack of actionable data. Since 2013, the amount of health care data has increased by nearly $50 \%$ per year.

Unfortunately, the vast quantity of data points generated, remain scattered, and therefore, their interpretation is timeconsuming and difficult, having tremendous impact on the quality of care. One small pilot study revealed that over $50 \%$ of patients in whom T2D coincided with hypertension and impaired kidney function were not receiving clinical guideline conform care for their hypertension.

\section{Integrated Personalized Diabetes Management}

In an effort to address clinical inertia, the EASD/ADA recently issued guidelines recommending a patient-centered, personalized approach to diabetes care to strengthen patient empowerment and the collaborative approach between the health care team and people with diabetes. The PDM-ProValue study, which evaluated exactly the feasibility of that concept, demonstrated that a structured personalized approach to diabetes management that puts the patient at the center of a circular care pathway based on shared decision-making and close integration of all essential stakeholders (referred to as Integrated Personalized Diabetes Management [iPDM]) improved clinical outcomes through earlier and more frequent therapy adjustments. ${ }^{40}$ A key driver of therapy intensification was increased patient adherence, which coincided with increased treatment satisfaction and improved patient/clinician interactions, both of which are known drivers for enhanced adherence. Importantly, clinicians reported increased satisfaction with the iPDM approach compared with their usual care delivery process, which is also a potential driver for the improved clinical outcomes observed.

\section{Digital solutions}

One approach to overcome the lack of oversight is targeted data aggregation and visualization, resulting in faster and more impactful therapy decisions (Accu-Chek Connect Reports Utility and Efficiency Study [ACCRUES]). 
As demonstrated in the PDM-ProValue study, the most promising health care applications provide digital solutions that efficiently gather and organize data automatically and present those data in formats that facilitate rapid assessment of patient status and support informed therapy decisionmaking. ${ }^{40,41}$ These solutions should also support collaboration and shared decision-making, empowering patients to become more knowledgeable and engaged in their treatment and enabling clinicians to identify and address patient obstacles to adherence.

Digital solutions that provide immediate availability of structured data, enhanced by decision support tools, facilitate greater collaboration and more informed shared decisionmaking. Importantly, digital solutions must integrate all aspects of diabetes patient care such as hypertension or dyslipidemia, which are often overlooked in daily clinical practice because of time constraints and challenges in accessing and/or assessing patient information during clinic visits.

These capabilities free up time for more meaningful patient/clinician interactions by automating the time-consuming processes previously performed manually by the clinicians during the face to face visits.

\section{Enhanced care through connectivity}

Although many available digital solutions remain closed systems that prohibit interoperability, Roche Diabetes Care has started to build an open ecosystem that will ultimately facilitate interconnectivity between various technologies from a wide range of device manufacturers and app/software developers. The goal is to minimize the gap between efficacy and effectiveness of care by informing physicians and to empower patients in a timely and effective manner.

\section{Summary}

Leveraging the new and exciting opportunities provided by digital transformation of the health care sector will revolutionize diabetes management by fostering shared decisionmaking of clinicians and patients and ensure the provision of effective and efficient routine care.

\section{Session D/Lecture 4}

\section{Predicting the Early Risk of Chronic Kidney Disease in People with Diabetes Using Real-World Data}

Wolfgang Petrich, Roche Diagnostics, Penzberg, Germany

\section{Overview}

Early medical risk assessment enables easier and more focused clinician/patient interactions and earlier, more targeted interventions, leading to improved clinical outcomes. Current treatment recommendations and risk stratifications are mostly based on tightly controlled randomized trials, often involving preselected patients and conducted under ideal conditions. As such, the outcomes from these studies do not necessarily reflect the effectiveness of interventions when applied to real-world populations.

Although the volume of real-world medical data from clinics and physician offices greatly exceeds the information available in clinical trials, the increase in data volume comes at the expense of completeness, uniformity, and control when using such RWD. Roche Diagnostics recently partnered with IBM to develop and evaluate an algorithm that more accurately assesses the medical risk for chronic kidney disease (CKD) compared with use of HbA1c and algorithms based on clinical trial data.

\section{Assessing the risk for CKD}

A recent study explored the use of RWD to identify individuals with diabetes who are at risk for developing CKD in the near future. ${ }^{42}$ The analysis compared an algorithm derived from patient records from 417,912 individuals with newly diagnosed T1D or T2D from the IBM Explorys database with algorithms derived from four large clinical trials. The Roche/IBM algorithm targeted seven features-age, $\mathrm{BMI}$, glomerular filtration rate, HbA1c, glucose, and concentrations of creatinine and albumin - as important predictors of risk based on data-driven and medical selection. The algorithm was applied to data originating from 104,504 further, independent individuals of the IBM Explorys database as well as from 82,912 individuals with T2D included in the Indiana Network for Patient Care (INPC) database.

Area under the receiver operating characteristic curve (AUC), a common measure of the quality of clinical markers, was used to evaluate the ability of the algorithms to correctly calculate the risk for developing CKD over the next 3 years. Using the seven prioritized measures, the AUC was $76 \%$. In the same population, HbA1c had an AUC of only $57 \%$. Investigators also assessed the prediction capabilities of the algorithm using subcohorts that matched the inclusion and exclusion criteria of the respective clinical studies. In this analysis, the AUC of the Roche/IBM algorithm remained superior to algorithms derived from the clinical trials.

Because some data out of the seven predictors might be unavailable in real-world clinical practice, investigators also explored the prediction capabilities of the algorithms when there are gaps in the data compared with the reference studies. Using the INPC database, investigators reported an AUC of $80 \%$ when all parameters are present, which remained relatively stable even if as few as $30 \%$ of the parameters were available. Further analysis showed that the mere size of cohort used to develop the algorithm is not the main reason for the superior performance of the Roche/IBM algorithm and it is speculated that the diversity of the training data has a larger impact than size.

\section{Summary}

The Roche/IBM algorithm outperforms algorithms published in the literature in a one-to-one comparison in a realworld cohort as well as in a subcohort analysis, demonstrating an AUC of $79 \%$ in predicting CKD development during the first 3 years subsequent to the initial diagnosis of diabetes. Importantly, accuracy is only slightly affected when fewer than the seven predictive factors used in the analyses are available to clinicians. These findings further fuel the debate on the efficacy and efficiency of using RWD compared with data derived from clinical trials. Moreover, they support the value of utilizing more RWD, potentially in combination with clinical trial data, for medical risk assessment. 


\section{Session E/Lecture 1}

\section{The PRO Solo Study: Setup and Hands-on Experiences with a New Micropump}

Julia Mader, Medical University of Graz, Graz, Austria

\section{Background}

Insulin pump use in adults and children has been increasing in recent years. This rise is mainly due to improvements in convenience, ease of use, and reimbursement. In addition, patients consider pumps to be a comfortable way of delivering insulin, resulting in improved adherence to the suggested bolus dose for snacks and when correcting slightly elevated glucose values. Because these bolus doses are often missed in pen users, insulin pump therapy-especially in combination with CGM-leads to improved glycemic control in the majority of patients.

\section{Patch pump versus conventional pump}

There are currently two types of insulin pumps on the market: conventional pumps that require an infusion set to deliver insulin under the skin; and patch pumps that are directly attached to the skin with an adhesive and deliver insulin via an integrated Teflon cannula. Patch pumps have several advantages over conventional pumps. The tubeless design eliminates the common problems associated with insulin infusion sets and reduces the risk of clogging. Many systems feature the ability to administer insulin manually and via a remote-control device. In addition, studies have shown that users found patch pump application to be less painful than infusion set insertion. The small size and multiple locations for patch pump placement on the body provide greater convenience and discretion.

However, there are also limitations that should be considered. For example, use of patch pumps often requires users to carry extra supplies when traveling. In addition, if filled at a higher level than required, any remaining insulin is wasted when a new pump is placed. Another disadvantage is that the insertion site is not visible, which increases the risk of undetected infections and cannula dislocations. Although many patch pumps are water resistant, they must be removed while swimming.

\section{Accu-Chek ${ }^{\circledR}$ Solo micropump system}

The most recent development in patch pumps is the AccuChek Solo micropump system, which obtained Conformité Européene (CE)-approval in 2018 and launched in selected markets. The system is currently being assessed in a multinational clinical trial in Europe.

The system uses a modular design comprising two main components that communicate wirelessly: the micropump and the Diabetes Manager (hand-held, remote control device). The micropump includes a pump base that can be used for up to 4 months with a disposable $2 \mathrm{~mL}$ (200 IU) insulin reservoir attached. The pump base houses the motor, electronics, memory, two bolus buttons, and the safety alert; all of which are found in conventional insulin pumps as well. The bolus buttons allow the user to deliver a bolus directly from the micropump without using the Diabetes Manager. The connection to the subcutaneous tissue and fixation to the body are ensured by an infusion assembly that is available with 6- or 9-mm cannulas. The modular design also allows to temporarily disconnect the micropump from the body (e.g., when exercising) as well as to replace individual system components as required.

\section{PRO Solo study}

The Accu-Chek Solo micropump system is currently being evaluated in the PRO Solo study, a prospective, multicenter, and multinational trial assessing the impact of patch pump use on treatment satisfaction and glycemia compared with MDI therapy in 180 individuals with T1D who are naive to insulin pump therapy. Participants are randomly assigned to the Accu-Chek Solo micropump system, Insulet OmniPod ${ }^{\circledR}$ insulin management system, or continued MDI therapy. At 6 months, all participants will be switched to the Accu-Chek Solo micropump system for an additional 13 weeks. The primary outcome measure is change in treatment satisfaction using the Diabetes Treatment Satisfaction Questionnaire (DTSQ). Secondary outcomes include device satisfaction, device performance, hypoglycemia, HbAlc, and material consumption.

As of April 2019, the study had enrolled 124 participants, 14 patients had completed the study and 8 participants discontinued; 1 in the Solo group. Preliminary feedback from participants has been positive. Most agreed that the Solo micropump is small, discrete, and easy to handle. The tubeless design and ability to manually deliver boluses were also highly rated features. However, not being waterproof and the inability to control the micropump with a smartphone were considered disadvantages.

\section{Requirements for switching from MDI to CSII}

Appropriate patients for insulin pumps are those who are motivated, have good knowledge about diabetes, and who have established self-management and problem-solving skills. Health care providers should be experienced in technology, specifically insulin pumps, and have a support team made up of a diabetologist, diabetes educator, and dietician who are familiar with both the system and reimbursement requirements. Good technical support from the manufacturer is also an important consideration.

The first step in initiating insulin pump therapy is to collaborate with patients and agree upon glycemic goals. The next step is to calculate the appropriate basal dose. If the patient is already using insulin therapy and is in good glycemic control, reducing the basal dose by $20 \%$ should be considered. If the patient is not in good control, a dose adjustment may be indicated. Age, body weight, and physical activity level are additional factors to consider when determining total daily dosages and in establishing time blocks. Importantly, glycemic changes are not apparent until $3 \mathrm{~h}$ after a change in basal insulin. Therefore, patients should be counseling to this time lag when using temporary rates. Temporary basal rates or profiles for situations that require lower rates (e.g., exercise and alcohol use) and higher rates (e.g., high-fat meal, stress, and illness) should also be calculated and set. Increases to $150 \%$ and reductions to $50 \%$ are generally considered good starting points.

The next step is determining bolus insulin requirements. The basal-to-bolus ratios commonly used are 30:70, but can 
vary also from 60:40, 40:60, and 50:50. When estimating bolus doses in patients with well-controlled diabetes, there is no need to change their current bolus doses. Doses can also be estimated by calculating the insulin-to-carbohydrate ratio $(350 \div$ Total Daily Dose for obese; $500 \div$ Total Daily Dose for lean) or insulin sensitivity factor (ISF) $(500 \div$ Total Daily Dose for obese; $2000 \div$ Total Daily Dose for lean).

The goal of diabetes management is to first avoid hypoglycemia, which is often a consequence of excessive glycemic variability due to overcorrecting or administering insulin too early when addressing high glucose, or by delivering the bolus too early. Patients should be well counseled regarding this issue and trained to use the bolus calculator, using defined time frames for insulin on board.

\section{Summary}

With proper training and accurate insulin parameters, insulin pump therapy can be used safely to achieve optimal glycemic control. Patch pumps present an effective and comfortable alternative to conventional insulin pumps.

\section{Session E/Lecture 2}

\section{Insertable Real-Time Continuous Glucose Monitoring at a New Level: Clinical Benefits of a 6-Month Sensor Wearing Time}

Dorothee Deiss, Medicover Berlin, Berlin, Germany

\section{Overview}

Use of CGM has been shown to improve HbA1c, increase time in target range, and reduce hypoglycemia. Despite the known benefits of CGM, most patients with T1D still do not use this technology, and usage in T2D is even lower. Moreover, adherence and persistence of CGM use are poor in many who have used this technology; $27 \%$ of patients who start CGM discontinue within 1 year due to several reasons.

\section{Unsolved issues with CGM systems}

Reasons for discontinuing CGM use include the burden of frequent, repeated insertions ( 25 to 50 times per year), fear of pain or discomfort, likelihood of accidental sensor dislocation, missed alarms, and the increasing number of serious skin reactions and allergies to adhesives. An ideal sensor would have a longer sensor life, require minimal insertions, and feature a transmitter that is easy to wear and remove as needed.

\section{Senseonics Eversense ${ }^{\circledR}$ real-time CGM system}

The Eversense real-time CGM (rtCGM) system (Senseonics, Inc., Germantown, MD) addresses the barriers to rtCGM use and may encourage more patients with diabetes to adopt CGM as a component of their self-management. The system is the only long-term CGM system currently available that has a fully implantable sensor lasting up to 180 days. Onbody vibration alerts are triggered if individualized high- and low-glucose thresholds are breached. The sensor is implanted in the upper arm. Users can remove the external transmitter for periods, for example, for discrete use or physical activity, without having to replace the sensor.
The system comprises three components: implantable sensor; "smart" transmitter; and mobile app. The sensor is implanted and removed during a minimally invasive, brief office-based procedure using customized insertion tools. The sensor technology is based on fluorescence, not electrochemistry, the technology used in other commercially available systems. Thus, there is no acetaminophen interference with the system. The sensor is powered by the small transmitter worn externally above the sensor. The transmitter is secured with a silicone-based adhesive that is changed daily for comfort. The mobile app runs on a smartphone or apple watch and provides real-time readings every $5 \mathrm{~min}$, glucose trends, and alerts, including predictive alerts. The Eversense ${ }^{\circledR}$ NOW app allows caregivers and health care providers to remotely view the patient's glucose information.

\section{Efficacy and safety}

The accuracy and safety of the Eversense system use has been demonstrated in both T1D and T2D over periods of 90180 days (masked and unmasked modes) in one pivotal trial in Europe ${ }^{43}$ and two trials conducted in the United States. ${ }^{4,45}$ The extensive data collected in the European PRECISE ${ }^{43}$ trial were used to further refine the algorithm accuracy, which was demonstrated to be comparable with other CGM systems $^{46}$ in the subsequent PRECISE $\mathrm{II}^{44}$ and PRECISION studies. ${ }^{45}$ Similar accuracy over 180 days was demonstrated in a primarily adolescent population. ${ }^{47}$ The new 602 algorithm, which improves the performance of sensor life, is already available in the United States and has been recently introduced in Europe.

In all of these studies, use of the Eversense system was demonstrated to be safe. Insertion and removal procedures and the device itself were well tolerated, with no adverse events related to insertion/removal or the device, itself. There were no infections, and only a few mild skin reactions to the sensor occurred. These fully resolved within 2 to 8 weeks of removal. Limited skin reactions were observed to the transmitter adhesive. The safety is further confirmed in the ongoing European registry of all Eversense users. Importantly, the reported adverse events were similar to those seen in other CGM systems.

\section{Germany experience}

Currently, there are more than 300 Eversense centers in Germany, with over 1800 patients using the system. Patients at the Berlin clinic have reported high satisfaction with the Eversense system, identifying extended longevity of sensor wear, consistent accuracy, and no sensor loss when removing the transmitter and no false alarms during the night as key benefits of the sensor. Users were also very satisfied with the removable transmitter, particularly the on-body alerts, which are triggered even when the smartphone is not nearby. Tape adherence and reduced skin irritations were also listed as positive features. Users also liked the app, which functions on both, the iOS and Android platforms, as well as the ability to input events (e.g., food intake and exercise) and to share the information with others. Although rare, preterm sensor failure and scarring at the insertion site were identified as concerns. Some patients were dissatisfied with the need to charge the transmitter batteries daily. 


\section{Patient selection criteria}

Patients most appropriate for the Eversense system are those who are experienced with smartphones and are consistent with smartphone use. In addition to all generally applicable CGM selection criteria, further specific characteristics are suggested to identify those patients who would benefit most from a long-term rtCGM: people who perceive frequent sensor replacement as a burden and those who are at high risk for disengaging an external sensor. History of skin problems, fear of pain, needle phobia, and the need for on-body alerts (e.g., drivers, athletes, and hearing impaired) are also indications for Eversense use.

\section{Summary}

The Eversense system offers significant advantages over other CGM systems. It is an important addition to the CGM landscape with the potential to expand CGM penetration, enabling more of patients to embrace CGM and achieve better glycemic control.

\section{Session E/Lecture 3}

\section{Automated Insulin Delivery and Decision Support Systems: From Science to Clinical Practice}

Jessica Castle, Oregon Health \& Science University, Portland, Oregon

\section{Overview}

Data from the T1D Exchange registry show that use of CGM has increased significantly over the past 8 years, from $6 \%$ to $38 \%$. The REPLACE-BG trial demonstrated that Dexcom CGM values can be used for making treatment decisions without confirmatory capillary blood glucose. The DIAMOND study, which included individuals with uncontrolled T1D and T2D treated with MDI, showed that the use of CGM improved HbA1c levels. However, despite this improvement, many participants had HbAlc levels above the typical goal of $<7 \%$. Can the vast amount of data provided by CGM be better leveraged to improve glucose control using decision support systems or automated insulin delivery (AID) systems?

\section{Current methods for insulin dosage calculation}

The current typical method for calculating mealtime insulin is based on planned carbohydrate intake, insulin-tocarbohydrate ratio (I:CHO), and the immediate glucose level compared with the glucose target. Similarly, correction dosages utilize the individual's ISF to calculate a dosage that will lower the current glucose to the target level. Insulin pumps offer integrated bolus advisors that automatically perform these calculations based on preset parameters (e.g., target glucose, I:CHO, and ISF) and patient inputted data (e.g., current glucose and carbohydrate intake). However, individuals treated with MDI therapy must perform these calculations manually (which increases risk of error) or rely on a smartphone app for bolus advice. Examples of these tools include the Accu-Chek ${ }^{\circledR}$ Connect app with bolus advisor, and the InPen ${ }^{\mathrm{TM}}$, a smart pen, which connects wirelessly to a smartphone app, collects insulin dose information, and then provides bolus advice.

\section{Decision support systems}

Although automated bolus advice capability clearly improves the accuracy of insulin dosage calculation, there are several limitations to current systems. For example, these systems use fixed settings that do not adjust to changes in insulin sensitivity, which is impacted by changing levels of physical activity, stress, illness, and other events of daily life. Decision support systems can be used to improve glucose control both by providing advanced on-demand bolus calculation and by automatically adjusting insulin dose settings over time. When combined with real-time CGM data, a decision support system could calculate both prandial and correction dosages based upon the direction and velocity of changing glucose (e.g., trend arrow data) and even facilitate "smart alarms"' that suppress false alerts and offer predictive hypoglycemia alerts based on the user's previous glucose trends, activity level, and meal data. A decision support system could also facilitate patient education and understanding. For example, if the system detects that the user is consistently bolusing too late or overtreating hypoglycemia, it could provide education at the time of the potential problem. There are currently multiple systems in development by TypeZero/Dexcom, Medtronic, Novo Nordisk, Lilly, Bigfoot, Sanofi, and Verily. Developers at Oregon Health and Science University are working on a decision support system that is currently being testing in a clinical trial. DreaMed has obtained FDA clearance and CE mark for its Advisor Pro, designed for individuals on insulin pump therapy. The Advisor Pro analyzes users' carbohydrate data and glucose data (CGM or blood glucose [BG]) and then automates the insulin pump settings. For example, if the system detects that an individual is consistently hyperglycemic overnight, it will recommend an increase in the overnight basal dosage.

\section{Automated insulin delivery}

AID is the current state-of-the-art for the treatment of T1D. Unlike traditional insulin pump therapy, AID systems automatically adjust insulin pump delivery rates based on incoming CGM glucose levels. As glucose levels rise, the insulin increases; when glucose levels fall, insulin is decreased or shut off. However, the success of these systems is impacted by the delayed onset and offset of current rapidacting insulin analogs. Slow onset of insulin can result in hyperglycemia following a meal; slow offset (up to $5 \mathrm{~h}$ ) can result in hypoglycemia even when insulin delivery is shut off. Weinzimer et al. showed that glucose levels were significantly better controlled when participants with T1D were given premeal insulin compared with fully AID. ${ }^{48}$ Most AID systems include some form of premeal insulin dosing to avoid postprandial hyperglycemia.

\section{MiniMed $^{\mathrm{TM}} 670 \mathrm{G}$}

The MiniMed 670G was the first AID system to be commercialized. The system requires mealtime announcements, requiring the user to manually enter planned carbohydrate intake. Although users have the option to administer correction doses manually, the correction factor is automated 
depending on how much insulin the individual has used over prior days. Otherwise, insulin delivery is automated based on the user's distance from glucose target, glucose area under the curve, glucose rate of change, and insulin-on-board safety constraints. The safety and efficacy of the $670 \mathrm{G}$ system when used in the closed-loop phase were demonstrated in a pivotal trial that showed significant reductions in $\mathrm{HbA1c}$, time in hypoglycemic ranges $(<70$ and $<50 \mathrm{mg} / \mathrm{dL})$, and time above target range $(>180 \mathrm{mg} / \mathrm{dL}){ }^{49}$

\section{Future AID systems}

The National Institutes of Health (NIH) has funded multiple large clinical trials to help further along the commercialization of other AID systems. For example, investigators are currently evaluating the safety and efficacy of the inControl system, which utilizes a model-predictive control system that anticipates the expected glucose response, determines whether the predicted response was correct, and then adjusts insulin delivery rates based on those data. Several other studies are underway, investigating systems using various components (e.g., insulin pumps and CGM devices) and controller algorithms.

\section{Summary}

AID and decision support systems are now feasible with current technology. Although meal and exercise announcements are still necessary at this time, these systems will likely require less and less input as these technologies evolve. The first AID system is now commercially available, and more are under development. Advances in these technologies have the potential to significantly improve glucose control and quality of life for people living with T1D.

\section{Session F/Keynote}

\section{Putting the Person with Diabetes in the Center: The New European Association for the Study of Diabetes/American Diabetes Association Consensus Report from 2018 on Management of Hyperglycemia in Type 2 Diabetes}

Richard Holt, University of Southampton, Southampton, United Kingdom

\section{The changing landscape}

Over the last decade, there have been tremendous innovations in diabetes medications and technology to help people with T2D manage their condition. Despite these developments, many people are not achieving their treatment goals. Much of suboptimal diabetes control is driven by barriers to accessing and utilizing successful diabetes treatments. The increasing complexity of medication regimens and the way many health care systems are organized have led to confusion and inertia among clinicians. Patient barriers, such as lack of education, cost issues, health beliefs, psychosocial obstacles, and numerous others, continue to impact patient selfmanagement behaviors. Another recent change in the diabetes landscape is the advent of CVOTs, which are impacting treatment decisions.

\section{Evolution of the EASD/ADA consensus}

The first iteration of the EASD/ADA consensus guidance for managing T2D was published in 2006. Since then, guidance in subsequent iterations has evolved from a strong focus on medication algorithms to a greater emphasis on the need to individualize all components of care, including appropriate use of medications, lifestyle interventions, and addressing the psychological barriers of diabetes.

The most recent consensus guidance, published in 2018, places greater emphasis on reducing clinical inertia and utilizing lifestyle interventions and medications that aim to improve both glycemic control and CV health. ${ }^{30}$ Importantly, the guidance includes a graphic depiction and discussion of the Decision Cycle for Patient-Centered Management, which places greater focus on patient-related issues that impact willingness and/or ability to follow treatment regimens.

\section{Assessing patient characteristics}

The decision cycle begins with an assessment of key patient characteristics (current lifestyle, comorbidities, and clinical characteristics) before initiating or adjusting therapy. For example, use of medications such as GLP-1 receptor agonists and SGLT-2 inhibitors should be considered for people with existing or at risk for $\mathrm{CV}$ disease and/or renal disease. However, the guidance is somewhat limited in identifying and explaining the psychological characteristics that should also be considered.

For example, although motivation of the person with diabetes is listed as a key characteristic, the drivers of motivation and its various manifestations are not fully explored. Some individuals may be motivated by external factors, such as being rewarded or avoiding punishment. Others are motivated by internal factors, such as the desire to achieve optimal health or personal satisfaction. Internal motivation is much more powerful than external motivation in changing behaviors.

Although ensuring that people with diabetes have the requisite knowledge and skills for effective diabetes selfmanagement, it is also important to understand how other factors, such as treatment satisfaction, health beliefs (positive and negative), and emotions, influence motivation and thus impact behavior change. It is well-established that individuals with diabetes have a twofold increased risk for depression, which can impact self-management behaviors and quality of life. However, other psychological disorders (diabetes-related distress, eating disorders, and anxiety) also impact self-management behaviors.

\section{Specific factors that impact choice of treatment}

Treatment goals must be individualized to address the key patient characteristics. For example, HbA1c targets should be balanced against a number of factors unique to each person with diabetes, such as weight gain, CV risk, hypoglycemia risk, complexity of regimen, medication side effects, disease burden, and others. Thus, medications that minimize weight gain, promote weight loss, reduce hypoglycemia risk, and effectively manage $\mathrm{CV}$ and renal disease should be considered. However, each individual's ability to access and afford these medications must also be considered. 


\section{Shared decision-making}

When determining a management plan, clinicians must put the person with diabetes at the center of care through shared decision-making. This is critical to supporting effective diabetes self-management during the $8758 \mathrm{~h}$ per year that they are not in contact with their health care professionals. Shared decision-making involves identifying patient preferences, feelings, and obstacles and then reaching mutual agreement on therapy and treatment goals. For example, rather than basing success on achieving $<7 \% \mathrm{HbA1c}$, a more reasonable goal for people with diabetes would be to take their medication daily or exercising regularly. Through this, people with diabetes will eventually reach optimal (or near) glycemic control through small steps that are more easily achieved and within their immediate control.

Importantly, clinicians must use these interactions to educate people with diabetes about the importance of their treatment regimen. Diabetes self-management education has been shown to be effective in lowering $\mathrm{HbA} 1 \mathrm{c}^{50}$ reducing all-cause mortality. ${ }^{51}$ Moreover, as demonstrated in the DAWN2 trial, participation in self-management program enhances quality of life, patient empowerment, and well-being. ${ }^{52}$ Unfortunately, fewer than $50 \%$ of people receive any education. ${ }^{52}$

\section{Summary}

The latest iteration of the consensus statement presents a much greater shift toward patient-centered care, with emphasis on the importance of shared decision-making and selfmanagement education. Moreover, it clearly highlights the need to individualize treatment with the understanding that not all therapies are equal.

\section{Session G/Lecture 1}

\section{The New World of Insulins}

Chantal Mathieu, University of Leuven, UZ Gasthuisberg, Leuven, Belgium

\section{Background}

People with T1D need exogenous insulin to maintain glucose homeostasis. In fasting states, basal insulin coverage is needed to maintain an anabolic state. Bolus insulin is needed at mealtimes to dispose the ingested glucose.

The availability of insulin analogs, with profiles that allow better coverage of mealtime glucose excursions and provide a more stable basal insulin coverage than previous human insulins, has allowed many people with T1D and T2D to achieve tighter glucose control with less risk of hypoglycemia.

However, despite advances in insulin formulations, current preparations still do not adequately mimic normal physiology. The onset of action of current mealtime insulins is too slow, failing to achieve the peak action that coincides with peak glucose excursions. With basal insulins, there is significant variability in their glucose-lowering effects, and many long-acting insulins do not last a full $24 \mathrm{~h}$. In addition, they must be administered at the same time every day, creating additional barriers to patient adherence. Mixing these insulins with other products remains problematic.

\section{New insulins in T1D}

Basal insulins. Among the newest basal insulins are the biosimilars, which are now being produced by the large manufacturers and many smaller companies around the world. Novel molecules (degludec) and more concentrated insulins (e.g., U300 glargine) are also being produced.

The advantage of degludec insulin is its stability and long duration. Although treatment with degludec showed similar reductions in HbA1c, fasting glucose, and confirmed hypoglycemia compared with glargine, significant reductions in nocturnal hypoglycemia have been demonstrated in earlier open-label studies. ${ }^{53,54}$ Hypoglycemia risk reduction has been confirmed in double-blinded, crossover studies. The disadvantage, however, is the higher cost. Importantly, because degludec is more potent than glargine, clinicians are cautioned to reduce the degludec dosage when transitioning patients from glargine.

More concentrated insulins, such as U200 degludec and U300 glargine, have recently been introduced. Although the pharmacokinetic/pharmacodynamic (PK/PD) of U200 and U100 degludec are identical, the PK/PD profiles of U100 glargine are notably different from the U300 formulation, which appears to be less potent. Therefore, when switching patients from U100 to U300 glargine, 10\%-15\% more insulin is needed. However, the advantages of U300 glargine-less hypoglycemia, better coverage, and more stable profiles - outweigh the cost of having to increase the dosage. This is particularly true in countries where the cost is less than the U100 preparation.

Mealtime insulins. Advances in mealtime insulin have led to a new generation of insulin preparations designed to provide a faster onset and shorter duration of action than current rapid-acting analogs. However, PK/PD studies reveal that the reduction in time to onset is only $\sim 5 \mathrm{~min}$. Moreover, clinical studies of these preparations have shown minimal HbA1c improvements, small differences in postprandial glucose excursions, and no difference in hypoglycemia. However, patients tend to favor these faster acting insulins because they reduce the waiting time between injecting the insulin and eating the meal.

\section{New insulins in T2D}

Current EASD/ADA guidelines now recommend using a GLP-1 agonist as the first injectable medication under most circumstances. The exception is when HbA1c levels are extremely elevated or when other symptoms (e.g., catabolism) are present. However, in patients with extensive (or complete) beta-cell failure, basal insulin is recommended because it improves glycemic control with minimal weight gain and hypoglycemia.

Clinical studies in T2D have demonstrated differences in hypoglycemia risk between the various basal insulins. For example, in insulin-naive T2D patients, treatment with U100 degludec over 2 years resulted in a $43 \%$ reduction in nocturnal hypoglycemia compared with U100 glargine. ${ }^{55}$ Similar findings have been reported in more recent trials. 
Regarding mealtime insulins, there appear to be no efficacy differences between the faster acting insulins and current rapid-acting analogs in T2D. However, manufacturers are now developing injectable preparations that combine basal insulin with GLP-1 receptor agonists. Two products are commercially available, IGlarLixi and IDegLira. These combinations provide HbA1c reductions similar to basalbolus therapy but with the added advantages of lower hypoglycemia risk and weight loss. As demonstrated in the DUAL VII study, treatment with IDegLira and metformin in patients with uncontrolled T2D resulted in $\mathrm{HbA} 1 \mathrm{c}$ reductions comparable with basal-bolus therapy, significantly lower hypoglycemia rates, and weight loss versus weight gain. ${ }^{56}$

\section{Summary}

New mechanisms of protraction have led to the development of novel basal insulins with profiles that provide stable basal insulin coverage with less hypoglycemia. Although the new faster on/faster off mealtime insulins provide some advantage over current rapid-acting analogs (e.g., convenience), improvements in clinical outcomes have been minimal. Combined preparations, such as IGlarLixi and IDegLira, show great potential in helping patients with T2D to safely achieve glycemic targets.

\section{Session G/Lecture 2}

\section{Noninsulin Drugs for People with Type 1 Diabetes: Is It Time for a Change?}

Francisco Javier Ampudia-Blasco, University Hospital Valencia, Valencia, Spain

\section{Background}

Data from the T1D Exchange registry show that a large proportion of individuals with T1D are not achieving their glycemic goals. This is particularly true in young adults. In addition, the prevalence of overweight/obesity in T1D is increasing. Since the Diabetes Control and Complications Trial (DCCT), intensive insulin therapy has been the treatment of choice in people with T1D. However, insulin therapy is frequently associated with hypoglycemia and weight gain. In addition, many patients cannot reach individual glycemic targets and/or have excessive glycemic variability despite strict adherence to their treatment. Since the DCCT, use of adjunctive therapies has been explored to mitigate these drawbacks.

\section{Metformin}

There are several benefits of using metformin in T1D, including improved insulin sensitivity, the ability to reduce insulin dosages, reductions in BMI, total and low-density lipoprotein (LDL) cholesterol, and a trend toward reducing HbA1c. However, gastrointestinal adverse effects, vitamin B12 deficiency, and increased hypoglycemia risk are limitations to its use. In the REMOVAL study, investigators assessed the $\mathrm{CV}$ and metabolic effects of metformin in T1D. ${ }^{57}$ Although reductions in weight were significant, only slight reductions in $\mathrm{HbA} 1 \mathrm{c}$ and no reductions in insulin dosages were seen. Importantly, there was no reduction in the progression of carotid artery intima-media thickness. In summary, the study findings do not support widespread use of metformin to improve glycemic control in adults with T1D.

\section{Pramlintide}

Pramlintide (available only in the United States) is a synthetic analog of amylin that lowers glucose through several mechanisms: it reduces appetite, slows gastric emptying, and decreases postprandial glucagon. In studies looking at the impact of diabetes duration $(7,16,30$ years) on the effects of pramlintide, HbA1c reductions were similar among age groups but with slightly greater reductions in weight and insulin dosages in the older populations. However, gastrointestinal side effects, increased hypoglycemia, and inconvenience of three daily injections limit its viability as an adjunctive therapy.

\section{GLP-1 receptor agonists}

GLP-1 receptor agonists have been shown to improve glycemic control and reduce insulin doses and weight. However, as shown in the ADJUNCT ONE trial, use of GLP1 receptor agonists is associated with increases in symptomatic hypoglycemia and ketosis episodes. Although this therapy may be beneficial for T1D patients who are overweight at diagnosis and have higher $\mathrm{C}$-peptide levels, its use is limited within the general T1D population.

\section{SGLT-2 and dual SGLT-1\&2 inhibitors}

SGLT inhibitors are a new class of medication. SGLT-2 inhibitors (empagliflozin, dapagliflozin, and canagliflozin) prevent reabsorption of glucose in the proximal tubules of the kidney by targeting SGLT-2, thereby increasing urinary glucose excretion. The only dual SGLT-1\&2 inhibitor, sotagliflozin, provides the added effect of delaying and reducing glucose absorption in the proximal intestine. Use of SGLT-2 and SGLT-1\&2 inhibitors is a well-established therapeutic option in T2D, and their insulin-independent mechanism makes these drugs an attractive option for adjunctive treatment of T1D.

Several phase 3 trials have consistently demonstrated reductions in HbA1c and glycemic variability and increased time in range without increased hypoglycemia for most patients treated with an SGLT-2 or SGLT-1\&2 inhibitor. 5,6,9,10 In addition, they are associated with weight loss and reduced insulin dosages.

The DEPICT- $1^{5}$ and DEPICT $-2^{6}$ trials (dapagliflozin) showed early and sustained decrease in HbA1c at 24 weeks, which was sustained over an additional 24 weeks, with no hypoglycemia and reductions in body weight. In these studies, both bolus and basal insulin dosages were reduced by $50 \%$. At daily dosages of $5 \mathrm{mg}$ and $10 \mathrm{mg}$, time in range increased significantly. Similar benefits were seen in the EASE study program (empagliflozin) but with increases in time in range at three of the dosages studied, 2.5, 10, and $25 \mathrm{mg} .{ }^{10}$ Results from the inTandem 2 study (sotagliflozin) were also similar. ${ }^{9}$

However, all studies showed an increase in DKA, which was due to a number of factors, including over-reduction in insulin dosages, infusion-site failure (insulin pumps), inconsistent carbohydrate intake, and vigorous exercise 
without taking appropriate precautions. These findings highlight the need for appropriate patient selection combined with thorough diabetes education to mitigate DKA risk.

\section{Summary}

Intensive insulin therapy with multiple daily injection and insulin pump is the mainstay for the treatment of T1D. However, intensive insulin therapy is also associated with hypoglycemia, weight gain, and glycemic variability. Adjunctive therapies may help to overcome these limitations in terms of further improving glycemic control with less hypoglycemia and weight gain. Despite some benefits, metformin, pramlintide, and GLP-1 receptor agonists are not viable options for these patients. Dapagliflozin and sotagliflozin were approved in Europe as adjunctive therapy in April and May 2019, respectively. More approvals are anticipated. However, selecting the right patient and preventing potential side effects by appropriate diabetes education are imperative.

\section{Session H/Lecture 1}

\section{Treating Diabetes in Canada}

Alice Cheng, Credit Valley Hospital in Mississauga, Toronto, Canada

\section{Background}

Canada is the world's second largest country by total area, but with a population of only 37 million people. The country is divided into 13 provinces and territories; $80 \%$ of the population lives in four of those provinces.

\section{Canadian health care system}

Canada utilizes a universal health care model that is federally funded but administered provincially. As mandated in Canada's constitution, access to health care services is guaranteed for all Canadians. Health care services are provided on three levels. Level 1 covers hospital/physician visits and diagnostic tests. Level 2 includes prescription drugs, home care, long-term care, and mental health treatment. Level 3 includes dental and vision care, complementary medicine, and outpatient physiotherapy.

Although all provinces provide full coverage for Level 1 services, coverage policies for Level 2 and Level 3 services and medications vary significantly from province to province. For example, individuals living in Ontario who are eligible may receive some of their medications free of charge, whereas individuals living on the east coast with the same criteria may have to pay out-of-pocket for the same medications. Health care delivery is challenged by ethnic diversity and varying levels of socioeconomic status within and between provinces. The large geographic area (with some communities accessible only by air travel) and uneven distribution of population pose additional obstacles. Canada must also address the significant disparities and social determinants of its indigenous population, which makes $4.3 \%$ of the total population. This group has higher rates of chronic disease, trauma, interpersonal and domestic violence, suicide, lower life expectancy, and infant mortality.
Although universal health care provides no-cost medical services to all Canadians, accessing care remains suboptimal. In recent study by Martin et al., investigators compared categories of access in Canada, the United States, the United Kingdom, France, Denmark, and Australia. They found notably higher proportions of Canadians who reported waiting $>2$ months for specialist appointments $(30 \%)$ and $>4$ months for elective surgery (18\%) versus U.S. wait times for specialists $(6 \%)$ and elective surgery $(4 \%){ }^{58}$ However, the percentage of patients reporting use of emergency department services due to lack of access to regular physicians was similar to U.S. percentages (17\% vs. $16 \%$, respectively).

\section{Diabetes in Canada}

More than 3 million Canadians, $8.1 \%$ of total population, have diabetes and another 8 million are living with prediabetes. The prevalence of both conditions is expected to rise. Interestingly, the highest prevalence of diabetes is found on the east coast, which is likely due to its geographic location where there are fewer health care professionals.

Aside from differences in provincial health care system structures, diabetes management is primarily informed by the Diabetes Canada clinical practice guidelines, which are published every 5 years. The most recent update was published in 2018. To improve access and the usefulness of these guidelines, Diabetes Canada created an interactive, webbased platform that provides decision support and screening tools, recommendations for glucose monitoring and medication regimens, and other relevant health information for both health care providers and patients. Despite these tools, the latest estimate of glycemic control in Canada suggests that about $50 \%$ of patients have an $\mathrm{HbA} 1 \mathrm{c} \leq 7 \%$; only $13 \%$ are achieving all three targets for glucose, blood pressure, and lipids.

\section{Summary}

Although universal health care is mandated for all Canadians, health care resource allocation and access differ from province to province. Within each province, medication access and availability also vary and are often dependent on age and/or financial needs. Diversity of geography, socioeconomic status, and ethnicity pose additional challenges to health care delivery. Unfortunately, little has changed in terms of $\mathrm{HbA} 1 \mathrm{c}$, blood pressure, and LDL status over the years. Although suboptimal diabetes control can be attributed to several factors, a novel approach to health care delivery is clearly required.

\section{Session H/Lecture 2}

\section{The Challenge of Diabetes in Southeast Asia and How Diabetes Is Managed in Singapore}

Sunil Sethi, National University Hospital, Singapore, Singapore

\section{Overview}

The Asia-Pacific region has $\sim 60 \%$ of the world population with tremendous ethnic and racial diversity. Approximately 300 million living in the region are older than 65 . The 
large, growing elderly population and increasing chronic disease load are creating significant challenges to health care systems. Many countries in this region lack the facilities, and health care providers needed to address the increasing prevalence of diabetes and other chronic diseases.

\section{Diabetes management in Singapore}

To address challenges of providing quality chronic care, Singapore divided the country into three segments. Each segment manages an ecosystem of $\sim 1.1-1.5$ million people, utilizing a comprehensive, longitudinal national electronic health record system that extracts and consolidates all clinically relevant information (e.g., illnesses, procedures, and adverse events) for every citizen from the time of birth into one record. This enables greater coordination and informed decision-making, resulting in more accurate diagnosis, better treatment, and integrated, patient-centric care.

Within the current system, patients are transitioned across a continuum of care as needed, from preventative chronic disease education and screening to definitive treatment and rehabilitation to end-of-life treatment. Every Singaporean can $\log$ in to his or her health data via the "HealthHub," which is a web-portal to review personal health appointments, immunization records, laboratory results, and other information. Financial assistance and counseling can also be accessed through the website, helping users to manage their health care costs.

\section{War on diabetes}

In 2018, Singapore began an initiative to address the challenges of diabetes based on more than 100 recommendations from a panel of 76 Singaporeans. Over a 6-week period, the panel listened to presentations from diabetes experts, formulated ideas, and then debated the merits of each idea. The Ministry of Health was then charged with determining the recommendations that would be immediately supported and those requiring additional consideration. Most recommendations were supported. The recommendations were divided into three main areas: education/awareness, healthy living/creating a supportive environment, and enhancing health care provider/caregiver skills.

\section{Role of the laboratory in supporting chronic disease management}

It is projected that in 2022, one in two diagnostic tests will be performed in point-of-care settings. Connected biosensors incorporated into clothing and implantable medical devices will be used to monitor vital signs, exercise, and nutrition, providing large quantities of data for mainframe analytics.

\section{Telehealth and decision support}

Telecommunication technologies are also being utilized to provide long-distance health care. The National University Hospital, Singapore, recently launched a telehealth initiative, utilizing four simple home devices: weighing scale, glucometer, blood pressure meter, and patient-international normalized ratio meter, which monitors patient response to warfarin. These devices, via a secure cloud, are connected through a $3 \mathrm{G}$ or $4 \mathrm{G}$ telecommunication network to the hospital. Data from patient measurements are reviewed by health care providers who either call patients or send emergency assistance, if needed, when a problem is detected. With this program, patients can continue with daily activities in addition to their usual testing, with assurance that a health care provider is monitoring the health and safety.

The laboratory team at the National University Hospital has also developed a decision support "dashboard" for diabetes management. The application pulls data from the patient's electronic medical record (EMR) and relevant information, particularly biomarker status, can be quickly reviewed and interpreted by the attending health care provider. Using a "traffic light" alert system, the dashboard identifies problem areas, such as high glucose and laboratory values that require immediate attention. The proof of concept is awaiting a live pilot trial once IT security and patient confidentiality details are managed.

\section{Summary}

Management of diabetes requires a holistic approach, with governments and nongovernment entities collaborating on strategies to address diabetes prevention and support provision of care at alternative locations. Exploitation and utilization of digital health care technologies will play a vital role in achieving these objectives.

\section{Session I/Keynote}

\section{Good Bugs and Bad Bugs: How the Intestinal Microbiome Contributes to Obesity and Diabetes}

\section{Stephan Bischoff, University of Hohenheim, Stuttgart, Germany}

\section{Background}

Within the past decade, almost 6000 studies of the human intestinal microbiome have been published. Approximately 1000 different species have been identified, expressing about 10 million genes - almost 500 times as many as the entire human genome. Although the function of the numerous bacterial genes is still largely unknown, various physiological and pathophysiological functions of the intestinal microbiome have been identified.

\section{The microbiome in health}

The gut microbiome is increasingly considered a potential physiological contributor to disease development and homeostasis. The physiological functions include immunomodulation and defense against pathogens and toxins, regulation of the central nervous system and enteric nervous system, energy homeostasis during cold conditions, and metabolic regulation, which is perhaps the most important influence.

Gut microbiota impact metabolic regulation in two ways. First, they provide key enzymes that allow humans to adapt quickly to new foods. Second, they enhance energy uptake from food, which is beneficial for those struggling with hunger but detrimental for those afflicted with obesity. This relationship was first learned from animal studies, which eventually led to investigations in humans within different geographic locations. For example, a 2010 study showed significant differences in the composition of intestinal Bacteroidetes in European children versus children from Burkina 
Faso (rural Africa). ${ }^{59}$ These differences were largely related to differences in foods, demonstrating that the microbiome always tries to adapt to diet to optimize digestion. An important regulator of gut microbiome is a group of foods now referred to as prebiotics. These are mostly fibers that cannot be digested in the small intestine but reach the large intestine where they are accessible as food for the microbiome.

\section{The microbiome in obesity}

The obesity epidemic is a growing concern. As such, there is increasing interest in understanding why humans gain weight differently eating the same diet and why obese individuals are afflicted by metabolic diseases such as diabetes and other conditions. Although low-fiber, high-protein, sugarrich diets (Western diet) are known to reduce the amount of microbiota-accessible carbohydrates, it was recently found that gut microbiomes differ between obese and nonobese individuals. These differences impact the way energy is utilized, expended, and stored. As the degree of obesity increases, the microbiome changes, resulting in dysbiosis. Dysbiosis is a condition characterized by increased hunger, fatty liver disease, inflammation, insulin resistance, and leaky gut. Interestingly, not all obese individuals develop dysbiosis, which suggests that it is dependent on the presence of a metabolic condition.

Another theory is that the metabolic disorders associated with dysbiosis may be caused by overnutrition (e.g., high sugar [fructose] load), which has been shown to sharply increase lipopolysaccharide levels, which can cause an increased expression of tumor necrosis factor alpha and inflammation in the gut. Persistent inflammation will result in liver steatosis.

An intact intestinal barrier protects the humans against invasion of microorganisms and toxins. However, this barrier must also be open to absorb essential fluids and nutrients. ${ }^{60}$ Recent studies have shown that fructose impairs the gut barrier. This impairment has implications not only for metabolic diseases but also for other conditions, such as depression and anxiety, frequent colds and food sensitivities, skin conditions, fatigue, colon disorders, rheumatoid arthritis, and fibromyalgia. Assessment of the lactulose/mannitol ratio, a measurement of gut barrier health, correlates with waist circumference, C-reactive protein, and all the markers of metabolic disease. There is also evidence of a second mechanism, resulting in translocation of bacterial endotoxin into the portal vein and then into the liver, causing a cascade of metabolic disorders. Most recently, investigators mechanistically linked hyperglycemia and intestinal barrier function with the systemic infectious and inflammatory consequences of obesity and diabetes. ${ }^{61}$

\section{The microbiome in T2D}

Individuals with T2D have depleted levels of butyrateproducing bacteria, which provide food for the cells lining the colon. A small randomized study of individuals with T2D treated with acarbose therapy showed that ingestion of dietary fiber enhanced butyrate production. ${ }^{62}$ Although acarbose, itself, is a microbiome modifier, subjects in the high-fiber group showed significant reductions in HbA1c levels. Another study investigated the effects of metformin therapy in treatmentnaive T2D patients. Investigators found that metformin had strong effects on the gut microbiome. Importantly, transfer of fecal samples from metformin-treated subjects to germ-free mice showed that glucose tolerance was improved in mice that received metformin-altered microbiota.

\section{Potential clinical consequences}

No definitive conclusions can be drawn from the small preclinical trials investigating the utility of microbiota interventions for prevention of secondary diseases. However, the potential is significant. For example, measurement of specific microbiomes could assist in assessment of disease risk and help in predicting an individual's response to therapy. Moreover, these measurements can also be used in conjunction with other data (blood tests, anthropometrics, and food diaries) to create a personalized nutrition predictor, which can be used to counsel patients about foods that cause excessive glucose responses.

Findings from current research suggest that future treatments, such as more targeted use of pre- and probiotics and fecal transplantation techniques, could potentially aid in the prevention of metabolic disease and possibly mitigate its associated complications. However, increased understanding of the relationship between microbiome status and diet provides impetus to more strongly focus on reducing sugar (particularly fructose) intake in daily life.

\section{Summary}

Diet is a major regulator of the gut microbiome. Overnutrition induces dysbiosis, causing enhanced energy harvest. High-sugar diets induce gut barrier dysfunction and low-grade inflammation. Fibers and other dietetic components can partially restore normal butyrate production. Antidiabetic drugs, such as metformin and acarbose, act, in part, through the gut microbiome. Microbiome analysis may facilitate disease risk assessment and prediction of dietetic effects. Microbiota therapy, using pro- and prebiotics, has the potential to provide new supportive antidiabetic medications.

\section{Session J/Lecture 1}

\section{The Science of Health Behavior Change}

\section{Paul Chadwick, University College London, London, United Kingdom}

\section{Background}

Behaviors are the most critical modifiable factors in both diabetes prevention and treatment adherence. However, behavior is also a factor in health care delivery. An estimated $40 \%$ of health care providers are not delivering evidencebased care and $\sim 5 \%-15 \%$ are delivering care that is harmful. Although most health care professionals recognize the importance of behavior, many efforts to address behavior lack behavioral science. Moreover, when the science is applied, it is often not given the same degree of attention and scrutiny as pharmacological interventions.

\section{Behavior Change Wheel}

The Behavior Change Wheel (BCW) is a standardized, integrative framework for designing and evaluating interventions to change behavior. ${ }^{63}$ It has recently been applied to 
diabetes. Development of the $\mathrm{BCW}$ was based on findings from a systematic search of electronic databases and consultations with behavior change experts to identify frameworks of behavior change interventions. The frameworks were evaluated according to their comprehensiveness, coherence, and having a clear link to an overarching model of behavior. The BCW utilizes the three key components of behavior change science: (1) a methodology for designing, delivering, and evaluating interventions; (2) a comprehensive theory of behavior; and (3) a replicable way of understanding what is inside complex behavior change interventions.

Methodology. The BCW methodology progresses through defined steps in the development, implementation, and evaluation of behavioral change interventions. The first steps are to define a specific outcome (e.g., improve HbA1c) and then identify all the behavioral determinants of that outcome. Identifying these determinants is particularly difficult because there are up to 63 separate behaviors involved in diabetes self-management, and each behavior must be identified in terms of who needs to do what differently, when, and how often. Moreover, it is important to understand how these behaviors are sequenced within routine and reactive selfregulatory cycles. For example, within a routine cycle, the behavior may be to adjust the insulin dose to accommodate carbohydrate intake. A reactive cycle would be to manage glucose levels in response to an event. Once the sequence of behaviors is understood, the intervention is designed, implemented, and evaluated. However, a theory is needed to understand the behavior. This will then drive the development of the intervention.

Comprehensive therapy of behavior. A recent systematic review identified 83 different theories of behavior in social and behavioral sciences. ${ }^{63}$ The University College of London Center for Behavior Change advocates the capability, opportunity, motivation and behavior (COM-B) system, which defines three necessary conditions for behavior: capability, motivation, and opportunity. ${ }^{63}$

Capability refers to both physical capabilities (e.g., injecting insulin) and psychological capabilities, such as having the requisite knowledge/skills and cognitive ability (e.g., adequate numeracy) to perform each behavior. Another component of psychological capability is self-regulation. Can the individual monitor his or her behavior over time? One aspect of psychological capability that is often overlooked involves interpersonal skills. Can the individual confidently and comfortably communicate with others regarding his or her self-management behaviors (e.g., performing blood glucose testing in public)?

Motivation can be categorized as reflective and automatic. Reflective motivation includes both an individual's belief about the potential consequences of engaging in the behavior and, importantly, whether the behavior is consistent with his or her personal identity. Automatic motivation relates to habits, such as eating (a habit difficult to change) and emotions, which often pose significant obstacles to engaging in desired health behaviors.

Opportunity refers to the physical and social factors that can influence behavior. Physical opportunity includes anything that directly enables or prevents performance of the behavior. For example, whether an individual has adequate time and tools needed to learn and consistently engage in the behavior. Social opportunity refers to the level of support an individual receives from his or her social network.

The BCW identifies nine main approaches that can be used to change behaviors, ${ }^{63}$ with predictable relationships between these approaches and how they influence specific behaviors. For example, if it is known that there is a deficit in a patient's knowledge/skills, then providing additional education would address that deficit. Whereas if cognitive ability is the issue (e.g., numeracy deficit), then use of enabling approach (e.g., bolus calculator app) would be appropriate.

However, achieving a desired behavior change requires policy decisions and other tools that mandate, incentivize, and support the various approaches. Examples include using the tax system to reduce individuals' out-of-pocket costs, establishing clinical guidelines for implementing behavior change interventions, greater utilization of psychologists in diabetes management, and utilizing media to reinforce healthy behaviors.

In summary, the BCW provides key advantages over other approaches. It is systematic, in that it is based on an extensive body of evidence (not just clinical intuition), considers all possible approaches to behavior change, and acknowledges that the environment has significant influence on behavior change. However, to replicate complex interventions, the scientific community needs to standardize the terms used to describe those interventions, evaluate the "active ingredients," and investigate the mechanisms of action within each intervention. This will facilitate synthesis of published reports into systematic reviews, which can be used to guide the development and use of these interventions in clinical practice.

\section{Summary}

Behavior is one of the crucial "missing links" in the ability to translate clinical and scientific knowledge into long-term positive outcomes for people with diabetes. Behavioral science can contribute to closing this gap, but only to the extent that it is based on a systematic approach to designing and evaluating behavior change interventions. Behavior change frameworks support the translation of science into practice. Good behavioral science looks at the way behaviors are influenced by the system, including the behavior of health care professionals.

\section{Session J/Lecture 2}

\section{Applying Self-Determination Theory in the Management of Type 1 Diabetes: The Role of the Health Care Team}

\section{Eveline Goethals, Joslin Diabetes Center at Harvard \\ Medical School, Boston, Massachusetts}

\section{Overview}

Treatment of T1D is intensive and demanding, requiring numerous daily activities. The emotional burden can be significant. In adolescents with T1D, the challenges of diabetes management are compounded by both physical and psychosocial developmental issues, such as the need to achieve a sense of identity, increasing autonomy in decision-making, reliance on peer groups for validation of 
self-concept, and integration of diabetes into their identity. Although many adolescent and young adults are able to balance these challenges, only $17 \%$ of adolescents and $21 \%$ of adults achieve the ADA goal of $<7 \% \mathrm{HbA} 1 \mathrm{c}$ due to poor treatment adherence. ${ }^{2}$

\section{Role of the health care team}

It is critical that diabetes health care teams identify actionable factors that can impact treatment adherence throughout the life span. With adolescents, the team needs to be mindful of the developmental changes and possible difficulties youths and their families may experience, while also identifying and supporting sources of strength. Signs of optimism and hopefulness, a supportive and warm parental environment, and peer support are requirements for the person's ability to meet the daily challenges and burden of diabetes. Importantly, the team should look for adaptive coping strategies.

Challenges for the health care team are often related to communication. Although difficult to achieve in busy clinics, it is important to make enough time for meaningful patient interactions. During those interactions, actively listening to the persons with diabetes and their family should be the priority; not only to what they say but also to what they do not say—or what they say between the lines.

\section{Self-determination theory}

The Self-Determination theory, ${ }^{64}$ an encompassing theory about human motivation, positions motivation across a continuum, moving from amotivation to extrinsic motivation toward intrinsic motivation. The goal is to move patients across the continuum to where their motivation is intrinsic-where patients are motivated to manage their diabetes because it aligns with their personal values. The health care team can help patients to move across the continuum by applying an autonomy-supportive communication style. This involves offering patients meaningful explanations and rationales for their treatment in ways that are personally relevant, and by providing choices, encouraging decision-making, and creating room for the patient's perspective within reasonable limits. Research on the specific role and value of the diabetes health care team in patients' motivation and treatment adherence across the life span is mounting.

\section{Current research}

A recent study by Goethals et al. examined the role of parental expectations and communication style toward adolescents' motivation to adhere to treatment guidelines for T1D. ${ }^{65}$ Structural equation modeling was used in a crosssectional, multi-informant study of 129 adolescents (mean age 14.43 years; $54.4 \%$ girls), 110 mothers, and 98 fathers. Adolescents reported on self-motivation, treatment adherence, and parental expectations and communication styles; parents reported on their own expectations, communication style, and perceptions of adolescent treatment adherence. The clarity of expectations regarding self-management tasks, as well as the manner parental expectations were communicated (controlling or autonomy supportive), was assessed. Medical record review provided $\mathrm{HbAlc}$ values.
Cross-sectional results showed that the quality of patients' motivation improved, with more internalization of diabetes guidelines and less rebellion, leading to greater treatment adherence and improved $\mathrm{HbA} 1 \mathrm{c}$ when adolescents perceived their parents to have clear expectations about the diabetes treatment and when parents express their expectations in an autonomy-supportive way. Conversely, motivation and adherence were negatively impacted when parents expressed their expectations in a controlling, punitive manner.

Another study by the same research group looked specifically at how adolescents perceived the autonomy support provided by their health care team in combination with autonomy support by parents. In this study, investigators utilized a validated questionnaire to assess whether patients felt that their health care team provided them choices and options, and whether the team tried to understand their perspectives on diabetes management before suggesting new approaches. Preliminary results showed that when patients had both a health care team and parents who were autonomy supportive, they showed better treatment adherence.

\section{Summary}

Use of the Self-Determination Theory offers a hands-on approach to patient-centered communication that highlights the personal relevance of diabetes treatment recommendations, values patients' perspectives, and allows for choice within reasonable limits (shared decision-making). The goal is to promote an atmosphere of openness, trust, and health information exchange. Moreover, the theory may lay the groundwork for future research.

\section{Session J/Lecture 3}

\section{Management of Type 1 Diabetes in the Digital Age: How Much Personal Interaction with Health Care Professionals Do We Still Need? A Review of the Evidence}

\author{
Jill Weissberg-Benchell, Ann \& Robert H. Lurie \\ Children's Hospital of Chicago, Chicago, Illinois
}

\section{Overview}

The current model of clinical diabetes care requires patients to attend periodic, face-to-face clinic visits. This requirement can be costly, time-consuming, and burdensome for patients and their families. The question is whether the use of current and future technologies can reduce this burden and, at the same time, enhance both the effectiveness and efficiency of patient/provider interactions, resulting in better clinical and psychosocial outcomes. Studies in use of mobile health (m-health) technologies suggest that the answer may be yes.

A growing number of people now have access to m-health technologies via smartphones and the Internet. Today, over 3.3 billion people own cell phones and $\sim 3.2$ billion have access to the Internet. In the United States, $95 \%$ of adolescents have a smartphone. Many program developers are leveraging the increasing access to communication technologies to create apps and web-based programs that could shift the current treatment paradigm to a new model of care. 
Text messaging, multimedia messaging, and m-health

Although most current approaches to utilizing m-health technologies involve text messaging to provide reminders to users, the most promising studies with texting involve texts that prompt the user to download data. The clinician then reviews the data and provides personalized feedback and advice to the patient.

In the recent SMS4BG study, adults with suboptimal diabetes control were provided a package of text messages personalized to their individual needs. ${ }^{66}$ The messages were designed to motivate participants to engage in health behaviors necessary for successful diabetes management. Participants could also choose to receive reminders for blood glucose monitoring, to which they could reply by sending in their result by text message. At 9 months, results showed significant reductions in $\mathrm{HbA} 1 \mathrm{c}$ compared with the control group, as well as improvements in health behaviors and perceived health status.

Multimedia messaging services offer even greater potential for improving both patient engagement and clinical outcomes. In the MD2ME study, adolescents and young adults were able to access web-based educational programs, receive text messages, and text with their health care providers to ask specific targeted questions. ${ }^{67}$ This intervention resulted in improved engagement in self-care behaviors, a greater sense of self-efficacy, and more patient-initiated communications compared with the control group.

\section{IDEAS framework}

The Integrate, Design, Assess, Share framework is a theoretical model for developing health apps. Development starts by integrating qualitative assessments of stakeholders to understand their needs/goals and identify and target specific behaviors for change, using strategies grounded in behavioral change theories. Interdisciplinary teams then work to develop and share prototypes to gather user feedback. The final program is then assessed, utilizing feasibility/acceptability studies, RCTs for efficacy, and real-world trials to measure effectiveness. The final step is to disseminate the program broadly.

\section{Health coaches/subscription services}

Another approach to utilizing m-health technologies uses health coaches via subscription services to improve outcomes; mySugr is an example. mySugr, an app-based program, offers two basic elements. The first element is a program that is free to download and enables users to log and track their glucose data, meal, exercise, and other activities. With the second element, users purchase a subscription that gives them access to health coaches and health care professionals. A free blood glucose meter and unlimited test strips are included in the service bundle.

Another company, Livongo, focuses on helping employers reallocate health care dollar resources by tracking employee health data (including glucose, blood pressure, and weight) and developing support systems. The Tidepool service offers users access to its data and provides feedback to both users and the health care providers. It is also possible for users to have their information deidentified for big data analyses in some cases.

\section{Telemedicine}

Use of telemedicine could reduce the burden of face-toface clinic visits (e.g., lost wages, time, and travel costs) and perhaps bridge the divide between high- and low-resourced families. A number of studies have shown that telemedicine visits confer equivalent or improved outcomes compared with the standard face-to-face care. Importantly, patient perceptions toward telemedicine visits are very positive. This approach may also result in cost savings for health care systems (e.g., lower facility costs, and fewer employees). However, large randomized trials are needed to demonstrate efficacy (clinical, psychological, and financial) followed by observational studies to demonstrate effectiveness in realworld settings.

\section{Summary}

Use of current and developing m-health technologies and telemedicine approaches has the potential to expand support for individuals with diabetes. These tools and approaches can be used to facilitate more comprehensive psychosocial screening, enhance learning, and support health care providers through greater connectivity to patient data and increased efficiency. However, optimizing implementation of these approaches requires significant changes within health care systems. Decision support tools are needed to enhance efficiency and make data actionable. Moreover, payers must be willing to cover all aspects of patient care, including data downloading and review, as well as clinician/patient interactions, conducted remotely and face-to-face.

\section{Session J/Lecture 4}

\section{The Opportunities of Digital Health: How Digital Coaching Is Successfully Delivering Lifestyle Change and Weight Loss for Patients}

\section{Rosie Carr and Michael Whitman, OurPath, London, United Kingdom}

T2D is a well-documented concern for Western health care systems, with a projected increase in prevalence and cost to health systems across the globe. Over 3 million people in England have been diagnosed with T2D; $90 \%$ of these individuals are overweight or obese. Obesity has been shown to increase the risk and progression of T2D, and it is risk factor for dyslipidemia, hypertension, CV disease, osteoarthritis, and cancer.

Obesity is driven by engrained lifestyle behaviors that impact and are impacted by several factors, such as food consumption, social and individual psychology, individual physiology, physical activity, and others. Fortunately, there is strong evidence that lifestyle interventions, focused on improving diet and increasing physical activity, can help people to lose weight and significantly improve their long-term diabetes outcomes.

Some research suggests that people who lose a significant amount of weight eventually regain the weight they lost or more; however, there is growing evidence that supports the effectiveness of digital behavioral change and lifestyle improvement programs in long-term weight control. Moreover, the effects of these approaches have been demonstrated to be comparable with in-person programs. 


\section{A multifactorial problem requires \\ a combinatorial solution}

People who are successful in losing weight and keeping it off are those who can persistently engage with the lifestyle advice they receive. However, behavior change is a slow process. One study showed that it takes an average of 66 days for a learned behavior to become automatic; however, the actual time can range from 24 to 254 days, depending on the individual. Therefore, ongoing support and advice are often needed. Although face-to-face counseling remains an effective approach, several barriers (e.g., time, location, and cost) prohibit frequent access to health care providers for needed advice, education, and encouragement.

Digital platforms can enable interventions that deliver information, education, and support via smartphones and website platforms, combining diabetes education with continuous encouragement remotely from dieticians and other members of the health care team. Digital coaching platforms can also increase cost-effectiveness for health systems, providing nutritional advice in an affordable and scalable setting while enhancing the efficiency and individualization of behavior change interventions.

\section{OurPath digital weight-loss program}

OurPath program is a digital lifestyle change program developed to support weight management and improve glycemic control in individuals with T2D. The 3-month program provides structured education, health tracking devices, and continuous support from health care providers and coaches. The program is designed to help users learn at their own pace, delivering information, training, and coaching while taking into account each individual's learning style, information/education needs, and level of support needed. In addition, the digital program links health tracking devices, which allow users to monitor their weight loss, physical activity, sleep, and other factors in real time. This continuous feedback reinforces the positive changes made, providing further motivation, which can lead to more positive outcomes and additional reinforcement. This ongoing monitoring also provides essential clinical feedback to dietitians, allowing them to tailor their nutritional advice, physical activity recommendations, and goal setting according to each user's progress.

After enrolling in the program, users with similar demographics, such as age, sex, and location, are placed into groups of up to 10 people. Each group is facilitated by a dietician or health coach. Group allocation algorithms automatically put similar people together, which can help to enhance user engagement. Communication is carried out via WhatsApp-style group chats. This provides an effective platform that allows patients to share experiences, suggestions, challenges, and questions Throughout the program, users progress through structured educational content, focusing on a variety of key healthy lifestyle components (e.g., exercise, sleep, nutrition, and positive thinking). The program app allows users to upload and record their food and drink intake throughout the day, which dietitians can review and then provide more individualized support through private chat.

\section{Effectiveness}

Continuous accessibility to dietitians has been shown to improve health outcomes. Although evidence is limited, early signs are promising. A recent trial showed $79 \%$ uptake from OurPath referral to registration. Users have experienced an $8.3 \%$ reduction in body weight at 6 months, and $40 \%$ of users reduced their $\mathrm{HbA} 1 \mathrm{c}$ to $<6.5 \%$.

Delivering a digital service provides contrast to typical avenues of dietetic interventions, such as a hospital ward or an outpatient clinic. These avenues of nutrition education can be particularly time and resource intensive. For example, dieticians may spend up to $1 \mathrm{~h}$ with a patient in a clinic and then see him or her again a few months later if at all. Through use of the OurPath digital health app, dietitians can coach up to 200 patients at once, educating and supporting them through lifestyle changes, providing more effective use of dietitians' time and other health care resources.

\section{Summary}

Independent economic analyses in the United Kingdom have shown that use of digital interventions can be cost effective, with cost savings of more than $£ 1000$ per patient after 5 years. These savings are realized from reductions in medications, fewer hospital admissions, and by preventing or delaying the development of future complications. Digital solutions have the potential to be valuable, cost-effective tools, allowing health care providers to reach large numbers of people to help prevent and better manage T2D throughout the world.

\section{Session K/Keynote}

\section{Radical Diabetes Prevention Programs: How Must They Be Set Up, What Can We Expect from Them, and What Is the Role of Health Information Technology?}

\section{Harpreet Sood, Regents Park Practice, London, United Kingdom}

\section{Background}

During the past 15 years, a growing body of evidence has demonstrated that diabetes can be prevented through intensive lifestyle interventions. The Diabetes Prevention Program, the most prominent of the major prevention trials, showed that individuals with impaired glucose tolerance who lost between $5 \%$ and $7 \%$ of body weight and participated in $150 \mathrm{~min}$ of moderate activity per week through a structured lifestyle program reduced their risk of developing diabetes by up to $58 \%$ over a follow-up of 2.8 years. ${ }^{68}$ The increasing number of people at risk for developing diabetes highlights the need for effective prevention initiatives.

\section{Health care is changing}

Globalization, an aging population, social media, rapid adoption of smartphones, increasing access to the Internet, and more sophisticated consumers are impacting health care and health care delivery. From a health system perspective, these changes are prompting a shift from "sickness-based" care to patient-centered disease prevention. There is also a 
shift from a paternalistic, data-controlled model to shared decision-making in which data are owned by the patient. Moreover, the workforce is moving from a manual laborbased paradigm to one that is driven by data, supported by technology that improves productivity, facilitating more flexible working.

In terms of diabetes, there are an increasing number of people at risk of developing the disease. Although projections show increasing prevalence in Europe and North America, the greatest increase is occurring in Southeast Asia and Western Pacific countries. This highlights the need for prevention programs that effectively address the needs of diverse populations. Current and emerging technologies will be useful in meeting these needs.

\section{National Health Service diabetes prevention program}

The U.K. National Health Service (NHS) launched a nation-wide diabetes prevention program in 2014. This evidence-based program draws upon large RCTs, which demonstrated that the incidence of T2D can be reduced by $30 \%-60 \%$ in at-risk patients (e.g., impaired fasting glucose and impaired glucose tolerance) through intensive lifestyle change interventions. At this time, the behavioral intervention is conducted in face-to-face interactions. The program has three goals: weight loss; achieving dietary recommendations; and achieving recommended levels of physical activity. The intervention is administered in 13 sessions, involving both group sessions and one-on-one meetings with a health coach who reviews each participant's progress, health, and well-being. Individuals referred to the program tend to have $6.0 \%-6.4 \% \mathrm{HbA} 1 \mathrm{c}$ values or fasting glucose of $100-125 \mathrm{mg} / \mathrm{dL}$. To date, 17,000 individuals have completed the program. Collectively, program completers have lost almost $60,000 \mathrm{~kg}$, an average of $\sim 3.4 \mathrm{~kg}$ per person.

The original goal of the NHS program was to have 100,000 referrals by 2020 ; however, increasing the goal to 200,000 referrals with 80,000 individuals enrolled in the program is being considered. Although this appears to be a large number, it is actually small, considering that more than 26 million people in the United Kingdom are at risk for diabetes and eligible coverage. Slow uptake may be due to logistical barriers; the program is only offered between 9 a.m. and 6 p.m., which is challenging for many people.

\section{Digital solutions}

Emerging evidence is demonstrating the effectiveness of using digital solutions to support behavior change programs. In July 2019, five organizations within the NHS launched an online version of the diabetes prevention program. This creates an opportunity for individuals to leverage digital technologies (e.g., wearable tracking devices and apps) to access health coaches and online peer support groups. Use of these technologies can accelerate uptake by improving user access and personalizing education content and coaching support. The new online program offers two-way communications, real-time feedback, and regular "check-ins" that keep users engaged. Online peer and social networks further enhance engagement and the user experience. From a health system perspective, these digital solutions allow health care providers and program administrators to collect data in real time and monitor the impact of the program.

Finding efficient ways to identify high-risk individuals, keeping them engaged, and integrating the program with other health initiatives remain key challenges moving forward. Moreover, pragmatic trials are needed to generate evidence for many of these digital solutions to better understand how they can be most effectively implemented and leveraged. However, recent data demonstrate that use of digital solutions for diabetes prevention improves outcomes and reduces health care costs. In the United States, cost savings from diabetes prevention initiatives utilizing digital technologies totaled over $\$ 7$ billion.

Because digital solutions require access to consumer data, strict guidelines are needed to protect personal information. The U.K. Department of Health recently published a code of conduct for data-driven health and care technology. This guidance includes a set of 10 principles that enable the development and adoption of save, ethical, and effective digital health care technologies.

\section{Sustainable solutions}

Accelerating the uptake and scalability of digital health solutions requires technologies that can be truly personalized to the individual needs/preferences of each user. It is also important to consider the changing role of the health care provider. There are some concerns that new technologies will replace or minimize the importance of health care providers. Therefore, it is important to communicate that adopting digital technologies for providing health coaching is not intended to replace jobs; rather, the goal is to enhance job performance so that patients receive higher quality care. Sustainability will require adoption of decision support technologies (e.g., artificial intelligence) that support lifestyle changes and more effective clinical management.

\section{Acknowledgments}

The authors thank all the presenters for their contributions.

\section{Author Disclosure Statement}

R.H. and C.Z. are employees of Roche Diabetes Care GmbH. C.G.P. has received consulting fees from Roche Diabetes Care GmbH. All presenters have approved the transcription of their presentations.

\section{Funding Information}

Funding for the development of this article was provided by Roche Diabetes Care GmbH, Mannheim, Germany.

\section{References}

1. Centers for Disease Control and Prevention (CDC): National Health and Nutrition Examination Survey, 2013-2016. ftp://ftp .cdc.gov/pub/Health_Statistics/NCHS/Dataset_Documentation/ NAMCS/doc2015.pdf (accessed July 12, 2019).

2. Foster NC, Beck RW, Miller KM, et al.: State of type 1 diabetes management and outcomes from the T1D Exchange in 2016-2018. Diabetes Technol Ther 2019;21:66-72. 
3. Carls G, Huynh J, Tuttle E, et al.: Achievement of glycated hemoglobin goals in the US remains unchanged through 2014. Diabetes Ther 2017;8:863-873.

4. Battelino T, Danne T, Bergenstal RM, et al.: Clinical targets for continuous glucose monitoring data interpretation: recommendations from the international consensus on time in range. Diabetes Care 2019;42:1593-1603.

5. Dandona P, Mathieu C, Phillip M, et al.: Efficacy and safety of dapagliflozin in patients with inadequately controlled type 1 diabetes: the DEPICT-1 52-week study. Diabetes Care 2018;41:2552-2559.

6. Mathieu M, Dandona P, Moshe P, et al.: Glucose variables in type 1 diabetes studies with dapagliflozin: pooled analysis of continuous glucose monitoring data from DEPICT-1 and 2. Diabetes Care 2019;42:1081-1087.

7. Garg SK, Henry RR, Banks P, et al.: Effects of sotagliflozin added to insulin in patients with type 1 diabetes. $\mathrm{N}$ Engl $\mathrm{J}$ Med 2017;377:2237-2348.

8. Buse JB, Garg SK, Rosenstock J: Fifty-two-week efficacy and safety of sotagliflozin, a dual SGLT and SGLT2 inhibitor, as adjunct therapy to insulin in adults with type 1 diabetes (in Tandem1). Diabetes Care 2018;67(Suppl 1). https://doi.org/10.2337/db18-212-OR (accessed September 20, 2018).

9. Danne T, Cariou B, Banks P, et al.: $\mathrm{HbA}(1 \mathrm{c})$ and hypoglycemia reductions at 24 and 52 weeks with sotagliflozin in combination with insulin in adults with type 1 diabetes: the European inTandem2 Study. Diabetes Care 2018;41: 1981-1990.

10. Rosenstock J, Marquard J, Laffel LM, et al.: Empagliflozin as adjunctive to insulin therapy in type 1 diabetes: The EASE Trials. Diabetes Care 2018;41:2560-2569.

11. Danne T, Garg S, Peters AL, et al.: International consensus on risk management of diabetic ketoacidosis in patients with type 1 diabetes treated with sodium-glucose cotransporter (SGLT) inhibitors. Diabetes Care 2019;42:1147-1154.

12. UK Prospective Diabetes Study (UKPDS) Group: Intensive blood-glucose control with sulphonlylureas or insulin compared with conventional treatment and risk of complications in patients with type 2 diabetes (UKPDS 33). Lancet 1998; 352:837-853.

13. ADVANCE Collaborative Group, Patel A, MacMahon S, et al.: Intensive blood glucose control and vascular outcomes in patients with type 2 diabetes. N Engl J Med 2008; 358:2560-2572.

14. The Action to Control Cardiovascular Risk in Diabetes Study Group: Effects of intensive glucose lowering in type 2 diabetes. N Engl J Med 2008;358:2545-2559.

15. Duckworth W, Moritz AC, Reda D, et al.: Glucose control and vascular complications in veterans with type 2 diabetes. N Engl J Med 2009;360:129-139.

16. Zoungas S, Patel A, Chalmers J, et al.: Severe hypoglycemia and risks of vascular events and death. N Engl J Med 2010;363:1410-1418.

17. O'Brien MJ, Karam SL, Wallia A, et al.: Association of second-line antidiabetic medications with cardiovascular events among insured adults with type 2 diabetes. JAMA Netw Open 2018;1:e186125.

18. Zinman B, Wanner C, Lachin JM, et al.: Empagliflozin, cardiovascular outcomes, and mortality in type 2 diabetes. N Engl J Med 2015;373:2117-2128.

19. Gæde P, Oellgaard J, Carstensen B, et al.: Years of life gained by multifactorial intervention in patients with type 2 diabetes mellitus and microalbuminuria: 21 years follow-up on the Steno-2 randomised trial. Diabetologia 2016;59: 2298-2307.

20. Eeg-Olofsson K, Zethelius B, Gudbjornsdottir S, et al.: Considerably decreased risk of cardiovascular disease with combined reductions in HbA1c, blood pressure and blood lipids in type 2 diabetes: report from the Swedish National Diabetes Register. Diab Vasc Dis Res 2016;13: 268-277.

21. Fatourechi MM, Kudva YC, Murad MH, et al.: Hypoglycemia with intensive insulin therapy: a systematic review and meta-analyses of randomized trials of continuous subcutaneous insulin infusion versus multiple daily injections. J Clin Endocr Metab 2009;94:729-740.

22. Benson K, Hartz AJ: A comparison of observational studies and randomized, controlled trials. N Engl J Med 2000;342: 1878-1886.

23. Ryder REJ, Sen-Gupta P, Thong KY; ABCD nationwide liraglutide audit contributors: Liraglutide is effective across a range of obese body mass indices; findings from the Association of British Clinical Diabetologists (ABCD) nationwide liraglutide audit. Diabetologia 2012;55[Suppl1]:S330 (Poster 801).

24. Dixon JB, O'Brien PE, Playfair J, et al.: Adjustable gastric banding and conventional therapy for type 2 diabetes: a randomized controlled trial. JAMA 2008;229: 316-323.

25. Lean M, Brosnahan N, McLoone P, et al.: Feasibility and indicative results from a 12-month low-energy liquid diet treatment and maintenance programme for severe obesity. Br J Gen Pract 2013;63:e115-e124.

26. Lean MEJ, Leslie WS, Barnes AC, et al.: Primary care-led weight management for remission of type 2 diabetes (DiRECT): an open-label, cluster-randomised trial. Lancet 2017;391:P541-P551.

27. Leslie WS, Ford I, Sattar N, et al.: The diabetes remission clinical trial (DiRECT): protocol for a cluster randomised trial. BMC Fam Pract 2016;17:20.

28. Nathan DM, Cleary PA, Backlund JY, et al.: Diabetes control and complications trial/epidemiology of diabetes interventions and complications (DCCT/EDIC) study research group: intensive diabetes treatment and cardiovascular disease in patients with type 1 diabetes. N Engl J Med 2005;353:2643-2653.

29. Ismail-Beigi $F$, Craven T, Banerji MA, et al.: Effect of intensive treatment of hyperglycaemia on microvascular outcomes in type 2 diabetes: an analysis of the ACCORD randomised trial. Lancet 2010;376:419-430.

30. Davies MJ, D'Alessio DA, Fradkin J, et al.: Management of hyperglycaemia in type 2 diabetes, 2018. A consensus report by the American Diabetes Association (ADA) and the European Association for the Study of Diabetes (EASD). Diabetes Care 2018;61:2461-2498.

31. Stone MA, Charpentier G, Doggen K, et al.: Quality of care of people with type 2 diabetes in eight European countries: findings from the guideline adherence to enhance care (GUIDANCE) study. Diabetes Care 2013;36: 2628-2638.

32. Gillies CL, Abrams KR, Lambert PC, et al.: Pharmacological and lifestyle interventions to prevent or delay type 2 diabetes in people with impaired glucose tolerance: systematic review and meta-analysis. BMJ 2007; 334:299. 
33. Dunkley AJ1, Bodicoat DH, Greaves CJ, et al.: Diabetes prevention in the real world: effectiveness of pragmatic lifestyle interventions for the prevention of type 2 diabetes and of the impact of adherence to guideline recommendations: a systematic review and meta-analysis. Diabetes Care 2014;37:922-933.

34. Khunti K, Wolden ML, Thorsted BL, et al.: Clinical inertia in people with type 2 diabetes: a retrospective cohort study of more than 80,000 people. Diabetes Care 2013;36:3411-3417.

35. Carls GS, Tuttle E, Tan RD, et al.: Understanding the gap between efficacy in randomized controlled trials and effectiveness in real-world use of GLP-1 RA and DPP-4 therapies in patients with type 2 diabetes. Diabetes Care 2017;40:1469-1478.

36. Khunti K, Seidu S, Kunutsor S, Davies M: Association between adherence to pharmacotherapy and outcomes in type 2 diabetes: a meta-analysis. Diabetes Care 2017;40:1588-1596.

37. Peyrot M, Rubin RR, Lauritzen T, et al.: Resistance to insulin therapy among patients and providers: results of the crossnational diabetes attitudes, wishes, and needs (DAWN) study. Diabetes Care 2005;28:2673-2679.

38. Zafar A, Stone MA, Davies MJ, Khunti K: Acknowledging and allocating responsibility for clinical inertia in the management of type 2 diabetes in primary care: a qualitative study. Diabet Med 2015;32:407-413.

39. Garcia-Perez LE, Alvarez M, Dilla T, et al.: Adherence to therapies in patients with type 2 diabetes. Diabetes Ther 2013;4:175-194.

40. Kulzer B, Daenschel W, Daenschel I, et al.: Integrated personalized diabetes management improves glycemic control in patients with insulin-treated type 2 diabetes: results of the PDM-ProValue study program. Diabetes Res Clin Pract 2018;144:200-212.

41. Hinnen DA, Buskirk A, Lyden M, et al.: Use of diabetes data management software reports by health care providers, patients with diabetes, and caregivers improves accuracy and efficiency of data analysis and interpretation compared with traditional logbook data: first results of the Accu-Chek Connect Reports Utility and Efficiency Study (ACCRUES). J Diabetes Sci Technol 2015;9:293-301.

42. Ravizza S, Huschto T, Adamov A, et al.: Predicting the early risk of chronic kidney disease in patients with diabetes using real-world data. Nat Med 2019;25:57-59.

43. Kropff J, Choudhary P, Neupane S, et al.: Accuracy and longevity of an implantable continuous glucose sensor in the PRECISE study: a 180-day, prospective, multicenter, pivotal trial. Diabetes Care 2017;40:63-68.

44. Christiansen MP, Klaff LJ, Brazg RR, et al.: A prospective multicenter evaluation of the accuracy of a novel implanted continuous glucose sensor: PRECISE II. Diabetes Technol Ther 2018;20:197-206.

45. Christiansen MP, Klaff LJ, Bailey TS, et al.: A Prospective multicenter evaluation of the accuracy and safety of an implanted continuous glucose sensor: the PRECISION study. Diabetes Technol Ther 2019;21:231-237.

46. U.S. Food and Drug Administration (FDA): Summary of safety and effectiveness data (SSED). Medical Device Database. http://www.fda.gov (accessed July 3, 2019).

47. Aronson R, Abitbol A, Tweden KS: First assessment of the performance of an implantable continuous glucose monitoring system through 180 days in a primarily adolescent population with type 1 diabetes. Diabetes Obes Metab 2019; 21:1689-1694.
48. Weinzimer SA, Steil GM, Swan KL, et al.: Fully automated closed-loop insulin delivery versus semiautomated hybrid control in pediatric patients with type 1 diabetes using an artificial pancreas. Diabetes Care 2008;31:934939.

49. Bergenstal RM, Garg S, Weinzimer SA, et al.: Safety of a hybrid closed-loop insulin delivery system in patients with type 1 diabetes. JAMA 2016;316:1407-1408.

50. Zhao FF, Suhonen R, Koskinen S, Leino-Kilpi H: Theory-based self-management educational interventions on patients with type 2 diabetes: a systematic review and meta-analysis of randomized controlled trials. J Adv Nurs 2017;73:812-833.

51. He X, Li J, Wang B, et al.: Diabetes self-management education reduces risk of all-cause mortality in type 2 diabetes patients: a systematic review and meta-analysis. Endocrine 2017;55:712-731.

52. Nicolucci A, Kovacs Burns K, Holt RI, et al.: Diabetes Attitudes, Wishes and Needs second study (DAWN2 ${ }^{\mathrm{TM}}$ ): cross-national benchmarking of diabetes-related psychosocial outcomes for people with diabetes. Diabet Med 2013; 30:767-777.

53. Heller S, Buse J, Fisher M, et al.: Insulin degludec, an ultralongacting basal insulin, versus insulin glargine in basal-bolus treatment with mealtime insulin aspart in type 1 diabetes (BEGIN Basal-Bolus Type 1): a phase 3, randomised, openlabel, treat-to-target non-inferiority trial. Lancet 2012;379: 1489-1497.

54. Bode BW, Buse JB, Fisher M, et al.: Insulin degludec improves glycaemic control with lower nocturnal hypoglycaemia risk than insulin glargine in basal-bolus treatment with mealtime insulin aspart in Type 1 diabetes (BEGIN( $(R)$ Basal-Bolus Type 1): 2-year results of a randomized clinical trial. Diabet Med 2013;30:1293-1297.

55. Zinman B, Philis-Tsimikas A, Cariou B, et al.: Insulin degludec versus insulin glargine in insulin-naive patients with type 2 diabetes: a 1-year, randomized, treat-to-target trial (BEGIN Once Long). Diabetes Care 2012;35:24642471.

56. Billings LK, Doshi A, Gouet D, et al.: Efficacy and safety of IDegLira versus basal-bolus insulin therapy in patients with type 2 diabetes uncontrolled on metformin and basal insulin: the DUAL VII Randomized Clinical Trial. Diabetes Care 2018;41:1009-1016.

57. Petrie JR, Chaturvedi N, Ford I: Cardiovascular and metabolic effects of metformin in patients with type 1 diabetes (REMOVAL): a double-blind, randomised, placebo-controlled trial. Lancet Diabetes Endocrinol 2017;5:597-609.

58. Martin D, Miller AP, Quesnel-Vallée A, et al.: Canada's universal health-care system: achieving its potential. Lancet 2018;391:1718-1735.

59. De Filippo C, Cavalieri D, Di Paola M, et al.: Impact of diet in shaping gut microbiota revealed by a comparative study in children from Europe and rural Africa. Proc Natl Acad Sci U S A 2010;107:14691-14696.

60. Bischoff SC, Barbara G, Buurman W, et al.: Intestinal permeability - a new target for disease prevention and therapy. BMC Gastroenterol 2014;14:189.

61. Thaiss CA, Levy M, Grosheva I, et al.: Hyperglycemia drives intestinal barrier dysfunction and risk for enteric infection. Science 2018;359:1376-1383.

62. Zhao L, Zhang F, Ding X, et al.: Gut bacteria selectively promoted by dietary fibers alleviate type 2 diabetes. Science 2018;359:1151-1156. 
63. Michie S, van Stralen MM, West R: The behaviour change wheel: a new method for characterising and designing behaviour change interventions. Implement Sci 2011;6:42.

64. Ryan RM, Deci EL: Self-Determination Theory: Basic Psychological Needs in Motivation, Development, and Wellness. New York, NY: Guilford Press, 2017.

65. Goethals ER, Soenens B, de Wit M, et al.: 'Let's talk about it' The role of parental communication in adolescents' motivation to adhere to treatment recommendations for type 1 diabetes. Pediatr Diabetes 2019;20: 1025-1034.

66. Dobson R, Whittaker R, Jiang Y, et al.: Effectiveness of text message based, diabetes self management support programme (SMS4BG): two arm, parallel randomised controlled trial. BMJ 2018;361:k1959.
67. Huang JS, Terrones L, Tompane T, et al.: Preparing adolescents with chronic disease for transition to adult care: a technology program. Pediatrics 2014;133:e1639-e1646.

68. Diabetes Prevention Program Research Group: Reduction in the incidence of type 2 diabetes with lifestyle intervention or metformin. N Engl J Med 2002;346:393-403.

Address correspondence to: Christopher G. Parkin, MS CGParkin Communications, Inc. 2352 Martinique Avenue Henderson, NV 89044

E-mail: chris@cgparkin.org 